\title{
A 2D Mathematical Model of Blood Flow and its Interactions in an Atherosclerotic Artery
}

\author{
S. Boujena ${ }^{1}, \mathrm{O}_{\mathrm{Kafi}^{1}}$, N. El Khatib ${ }^{2 *}$ \\ ${ }^{1}$ Université Hassan II-Casablanca, Faculté des Sciences -Ain Chock-, B.P 5366. Maarif. Casablanca \\ ${ }^{2}$ Department of Computer Science and Mathematics, Lebanese American University - Byblos campus \\ P.O. Box: 36, Byblos, Lebanon
}

\begin{abstract}
A stenosis is the narrowing of the artery, this narrowing is usually the result of the formation of an atheromatous plaque infiltrating gradually the artery wall, forming a bump in the ductus arteriosus. This arterial lesion falls within the general context of atherosclerotic arterial disease that can affect the carotid arteries, but also the arteries of the heart (coronary), arteries of the legs (PAD), the renal arteries... It can cause a stroke (hemiplegia, transient paralysis of a limb, speech disorder, sailing before the eye). In this paper we study the bloodplaque and blood-wall interactions using a fluid-structure interaction model. We first propose a 2D analytical study of the generalized Navier-Stokes equations to prove the existence of a weak solution for incompressible non-Newtonian fluids with non standard boundary conditions. Then, coupled, based on the results of the theoretical study approach is given. And to form a realistic model, with high accuracy, additional conditions due to fluid-structure coupling are proposed on the border undergoing inetraction. This coupled model includes (a) a fluid model, where blood is modeled as an incompressible non-Newtonian viscous fluid, (b) a solid model, where the arterial wall and atherosclerotic plaque will be treated as non linear hyperelastic solids, and (c) a fluidstructure interaction (FSI) model where interactions between the fluid (blood) and structures (the arterial wall and atheromatous plaque) are conducted by an Arbitrary Lagrangian Eulerian (ALE) method that allows accurate fluid-structure coupling.
\end{abstract}

Keywords and phrases: stenosed artery, atheromatous plaque, blood flow, non-Newtonian fluid, fluid-structure interaction, generalized Navier-Stokes equations, boundary conditions

Mathematics Subject Classification: 35Q30, 92C50, 74F10

\section{Introduction}

Blood has a quadruple role: the transport of chemical species to target organs, the regulation of temperature, $\mathrm{pH}$ and electrolyte fluid balance, defense against foreign bodies, and the prevention of bleeding by clotting. The third function involves, in particular, the docking of lymphocytes on the wet face of the vascular endothelium and then the crossing of the wall[4]. A similar phenomenon participates in the formation of atherosclerotic plaques. The initial stage of atherogenesis is characterized by the accumulation of lipoproteins in the subendothelial layer, when the inflow is not compensated by the outflow.

${ }^{*}$ Corresponding author. E-mail: nader.elkhatib@lau.edu.lb 
These molecules are, then, captured by circulating macrophages, attracted to the wall, which will form foam cells: Figure 1. Mass transport in the arterial lumen of physiological vessels, deposit of particles convected to the wall and their transfer through the wall, can not be treated simultaneously, because of the complexity of each process. In fluid mechanics, the investigation is focused on places of deposit, in the light of possible values of local shear stress. Convected particle deposition are, indeed, preferentially located in regions where the velocity gradient at the wall is weak, i.e. when the residence time near the wall is longer, as, for example, on the border of a detachment. Thus, the mechanism dependent on the shear rate is based on the mass transport between the blood and the wall, unlike a process induced by shear stress, where the direct action of forces on the wall is involved. However, the latter mechanism may affect the deposition of convected particles.

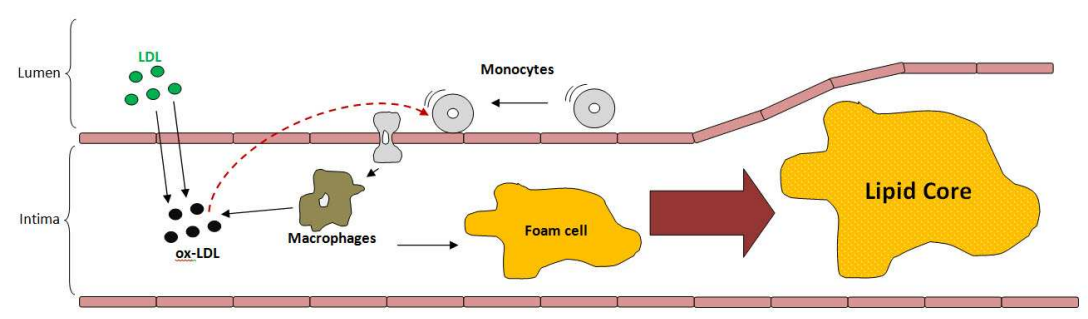

FIgURE 1. Schematic representation of the atherogenesis. The LDL penetrates the intima of the blood vessel where it is oxidized. The OX-LDL triggers the recruitment of the monocytes. The monocytes penetrate the intima and transform into macrophages which phagocyte the OX-LDL leading then to the formation of the foam cell and then to the lipid core that changes the geometry of the blood vessel by narrowing it.

Acute cerebral ischemic events are associated with rupture of vulnerable carotid atheroma: Figure 2 and thus thrombus formation. Stenosis and fibrous cap thickness have been considered as important risk factors for plaque rupture. A model of fluid-structure interaction to simulate the interaction between blood flow and atherosclerotic plaque has been proposed, to assess the effect of the degree of stenosis and fibrous cap thickness on vulnerable plaque, the fluid is assumed laminar, Newtonian and incompressible [18]. Another study was based on the same model, with a non-Newtonian fluid but both models assumed that the vessel wall is rigid [11].

On the other hand, it is well known that arteries are not rigid: they deform under the effect of blood flow and, naturally, their deformation in turn influences the flow. As in [7] which is concerned with the coupling of a generalized Newtonian fluid, accounting for the shearthinning behaviour of blood, with an elastic structure describing the vessel wall, to capture the pulse wave due to the interaction between blood and the vessel wall in a 3D case of a healthy artery. This interaction can be modeled roughly or neglected to address certain issues. This is the case for "clinical" problems. However, if it is to evaluate mechanical stresses distributed on the walls of vessels or stents, as in typical problems of "bio-engineering", while taking into account the mechanical interaction between blood and wall becomes important. However, this coupled problem is particularly difficult to solve numerically. It appeared in the late 90 s that the coupling algorithms used successfully in aeroelasticity (weak coupling, i.e. does not preserve the energy balance) were unstable for blood flow. This motivated the development of many methods of strong coupling, by nature extremely costly and complex.

The different sections of this work are presented as follow. In section 2 we introduce the generalized Navier-Stokes equations reformulated to have the non-Newtonian behavior. Being a non standard formulation, we carry out a well posedness analysis, providing an existence result for the evolutionary case, under suitable hypothesis on the boundary conditions. In section 3 we describe the structure model. We choose the St. Venant-Kirchhoff model which is a generalization of the linear model for large displace- 


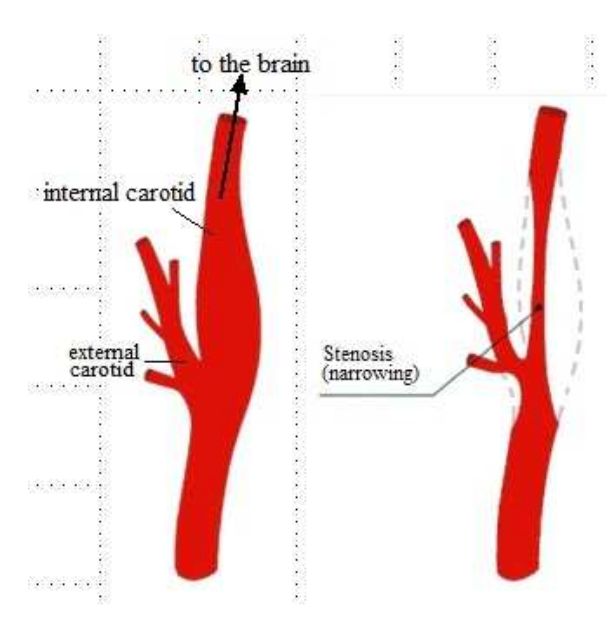

Figure 2. Stenosis of the internal carotid

ments, and is a particular case of hyperelastic materials. In section 4 we recall the coupling conditions between the adopted model of the fluid equations and the model for the movement of the structures. Numerical and physical parameters are presented in section 5 used for simulations shown in section 6 to illustrate the differences between the Newtonian model and the non-Newtonian Carreau model in the case of fixed and moving wall.

\section{The Fluid Equations}

In most part of the arterial system of healthy individuals blood can be modelled as a Newtonian fluid. However, in some disease states, namely if the arterial geometry has been altered to include regions of recirculation, detected for instance in intracranial aneurysms or downstream of a stenosis, more complex blood constitutive models should be used, although many authors have modelled blood flow using the Navier-Stokes equations. Therefore, we must develop rheological models as close as possible to reality to define the viscosity as a function of the tensor $\mathbf{D u}=\frac{1}{2}\left(\nabla \mathbf{u}+\nabla^{T} \mathbf{u}\right)$, or more precisely as a function of $\mathbf{s}(\mathbf{u})$, the second invariant of the strain rate tensor, defined by:

$$
(\mathbf{s}(\mathbf{u}))^{2}=2 \mathbf{D u}: \mathbf{D u}=2 \sum_{i, j}(\mathbf{D u})_{i j}(\mathbf{D u})_{j i}
$$

which appears in the constitutive law for an incompressible fluid after that given by Newton in 1687 for what is called newtonian fluid, which can be written

$$
\sigma(\mathbf{u}, p)=2 \mu \mathbf{D u}-p \mathbf{I}
$$

where $p$ denotes the pressure, $\mathbf{u}$ the velocity and $\mu$ the viscosity (constant) coefficiant. It is a linear law that was long believed satisfied for all fluids. But by the evolution of industrial techniques, it was found that the behavior of some synthetic mixtures did not obey the law (2.1). Fluids do not obey the law (2.1) are the class of non-Newtonian fluids for which we introduced a new constitutive law [3]

$$
\sigma(\mathbf{u}, p)+p \mathbf{I} \in \partial \varphi(\mathbf{D u})
$$

where $\varphi$ is a lower semi-continuous convex functional depending on $\mathbf{u}$ only through the second invariant of $\mathbf{D u}, \varphi:\left(L^{2}(\Omega)\right)^{\mathbf{n} \times \mathbf{n}} \longrightarrow \mathbb{R}, \Omega$ is an open bounded domain of $\mathbb{R}^{\mathbf{n}}(\mathbf{n}=2$ or $\mathbf{n}=3), \partial \varphi(\mathbf{D u})$ denotes the subdifferential of $\varphi$ at the point $\mathrm{Du}$ :

$$
\partial \varphi(\mathbf{D u})=\left\{\Phi \in X^{\prime} ; \varphi(\mathbf{D v})-\varphi(\mathbf{D u}) \geq(\Phi, \mathbf{D v}-\mathbf{D u}) ; \forall \mathbf{v} \in L^{2}(\Omega)\right\}
$$


with $X=\left(L^{2}(\Omega)\right)^{\mathbf{n} \times \mathbf{n}}$. $\sigma(\mathbf{u}, p)+p \mathbf{I}$ is denoted by $\sigma^{\mathbf{D}}$. Let $\bar{\mu}$ be a lower semi-continuous differentiable convex real function such that

$$
\varphi(\mathbf{u})=\int_{\Omega} \bar{\mu}(\mathbf{D u}) d x
$$

If $\varphi$ is differentiable, then the constitutive law (2.2) can be written:

$$
\sigma(\mathbf{u}, p)=-p \mathbf{I}+2 \mu(\mathbf{s}(\mathbf{u})) \mathbf{D u} .
$$

Fluids whose behavior is governed by (2.3) are called generalized Newtonian fluids. In fact, it is indeed a non-Newtonian constitutive law.

Given the universal laws, we obtain for the generalized Newtonian fluids the equations of motion (2.4) and (2.5) which constitute the generalized Navier-Stokes problem and coincide with the Navier-Stokes problem when the viscosity $\mu$ is constant.

The purpose of the following section is to demonstrate the existence of a weak solution to the generalized Navier-Stokes problem for a particular class of non-Newtonian fluids whose behavior is governed by $(2.2)$.

\subsection{Mathematical Model}

We consider a domain $\Omega_{f}$ where there is blood flow in a longitudinal section of a stenosed artery. Let $\Omega_{f}$ be an open bounded domain of $\mathbb{R}^{2}$ representing a portion of a cylindrical deseased artery. We denote by $\left(\Gamma_{w_{i}}\right)_{i=1,2}$ the portion of the boundary corresponding to the physical arterial wall, while $\Gamma_{\text {in }}$ and $\Gamma_{\text {out }}$ represent the so called artificial boundaries, since they do not correspond to any physical interface and $\partial \Omega_{f}=\bar{\Gamma}_{\text {in }} \cup \bar{\Gamma}_{w_{1}} \cup \bar{\Gamma}_{w_{2}} \cup \bar{\Gamma}_{\text {out }}$ with $\Gamma_{\text {in }} \cap \Gamma_{w_{1}} \cap \Gamma_{w_{2}} \cap \Gamma_{\text {out }}=\emptyset$ : Figure 3 .

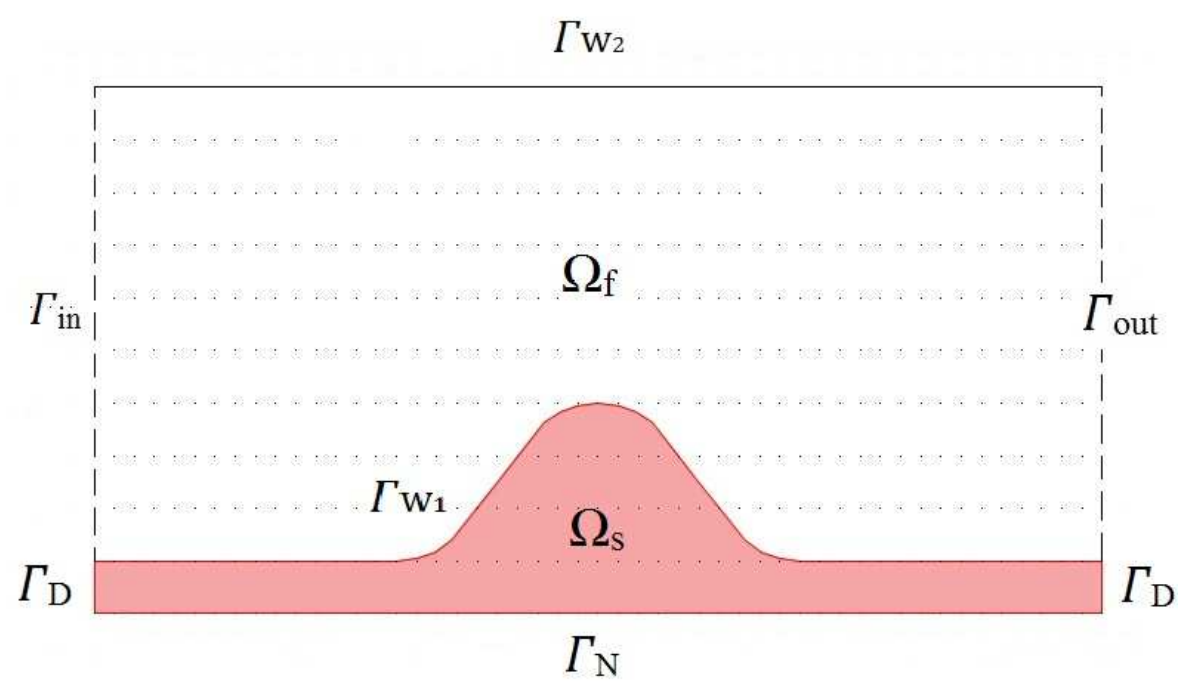

FiguRE 3. Geometric configuration

We will ignore body forces, both for the fluid and the structures in the next sections. For Haemodynamic applications this correspond in practice to ignore the effects of gravity. 
The evolution problem involves a velocity $\mathbf{u}=\left(\mathbf{u}_{1}, \mathbf{u}_{2}\right)$ and a pressure $p$ defined over $\Omega_{f} \times(0, T)$ such that

$$
\begin{gathered}
\rho_{f} \frac{\partial \mathbf{u}}{\partial t}+\rho_{f}(\mathbf{u} \cdot \nabla) \mathbf{u}-\nabla \cdot(2 \mu(\mathbf{s}(\mathbf{u})) \mathbf{D u})+\nabla p=0, \text { in } \Omega_{f} \times(0, T), \\
\nabla \cdot \mathbf{u}=0, \text { in } \Omega_{f} \times(0, T) .
\end{gathered}
$$

These equations are complemented with the boundary conditions over the boundary of $\Omega_{f}$

$$
\begin{gathered}
2 \mu(\mathbf{s}(\mathbf{u})) \mathbf{D u} \cdot \mathbf{n}-p \mathbf{n}=\mathbf{h}, \text { on } \Gamma_{\text {in }} \times(0, T), \\
2 \mu(\mathbf{s}(\mathbf{u})) \mathbf{D u} \cdot \mathbf{n}-p \mathbf{n}=0, \text { on } \Gamma_{\text {out }} \times(0, T), \\
\mathbf{u} \cdot \mathbf{n}=0 \text { and } 2 \mu(\mathbf{s}(\mathbf{u})) \mathbf{D u} \cdot \mathbf{n}-p \mathbf{n}=\mathbf{g}, \text { on } \Gamma_{w_{1}} \times(0, T), \\
\mathbf{u}=0, \text { on } \Gamma_{w_{2}} \times(0, T),
\end{gathered}
$$

together with the initial condition

$$
\mathbf{u}=\mathbf{u}_{0}, \text { for } t=0 \text { in } \Omega_{f},
$$

with $\mathbf{n}$ the outward normal vector.

In this section we will prove the existence of a weak solution for the problem (2.4)-(2.10) in the case of a non-Newtonian flow.

Let us introduce the following functional spaces:

$$
\mathcal{V}=\left\{\varphi \in\left(C^{\infty}\left(\overline{\Omega_{f}}\right)\right)^{2} \mid \nabla \cdot \varphi=0, \varphi \cdot \mathbf{n}=0 \text { on } \Gamma_{w_{1}}, \varphi=0 \text { on } \Gamma_{w_{2}}\right\}
$$

and

$$
V=\overline{\mathcal{V}}^{\left(H^{1}\left(\Omega_{f}\right)\right)^{2}}
$$

equiped with the scalar product as follows:

$$
((\mathbf{u}, \mathbf{v}))=\int_{\Omega_{f}} \mathbf{D u}: \mathbf{D} \mathbf{v} d x=\frac{1}{4} \sum_{i, j=1}^{2} \int_{\Omega_{f}}\left(\frac{\partial \mathbf{u}_{i}}{\partial x_{j}}+\frac{\partial \mathbf{u}_{j}}{\partial x_{i}}\right)\left(\frac{\partial \mathbf{v}_{i}}{\partial x_{j}}+\frac{\partial \mathbf{v}_{j}}{\partial x_{i}}\right) d x .
$$

The associated norm is denoted $\|\cdot\|$. It is clear that

$$
V=\left\{\mathbf{v} \in\left(H^{1}\left(\Omega_{f}\right)\right)^{2} \mid \nabla \cdot \mathbf{v}=0 \text { in } \Omega_{f}, \mathbf{v} \cdot \mathbf{n}=0 \text { on } \Gamma_{w_{1}}, \mathbf{v}=0 \text { on } \Gamma_{w_{2}}\right\}
$$

is closed subspace of $\left(H^{1}\left(\Omega_{f}\right)\right)^{2}$, so it is Hilbert space.

We remind that the scalar product of $\left(L^{2}\left(\Omega_{f}\right)\right)^{2}$ is denoted by $(, \quad)$, with the associated norm $|\cdot|$, such that for $(\mathbf{u}, \mathbf{v}) \in\left(L^{2}\left(\Omega_{f}\right)\right)^{2}$ we have:

$$
(\mathbf{u}, \mathbf{v})=\sum_{i=1}^{2} \int_{\Omega_{f}} \mathbf{u}_{i} \mathbf{v}_{i} d x
$$

Multiplying (2.4) by a test function $\mathbf{v} \in \mathcal{V}$ and integrating by using Green formula and the boundary conditions (2.6)-(2.9) we obtain

$$
\rho_{f} \int_{\Omega_{f}} \frac{\partial \mathbf{u}}{\partial t} \mathbf{v} d x+(A \mathbf{u}, \mathbf{v})+\rho_{f} b(\mathbf{u}, \mathbf{u}, \mathbf{v})=\int_{\Gamma_{i n}} \mathbf{h v} d \sigma+\int_{\Gamma_{w_{1}}} \mathbf{g v} d \sigma \forall \mathbf{v} \in \mathcal{V}
$$


where

$$
\begin{gathered}
(A \mathbf{v}, \mathbf{w})=\int_{\Omega_{f}} 2 \mu(\mathbf{s}(\mathbf{v})) \mathbf{D} \mathbf{v}: \mathbf{D} \mathbf{w} d x \\
b(\mathbf{u}, \mathbf{v}, \mathbf{w})=\sum_{i, j=1}^{2} \int_{\Omega_{f}} \mathbf{u}_{i} \frac{\partial \mathbf{v}_{j}}{\partial x_{i}} \mathbf{w}_{j} d x .
\end{gathered}
$$

A weak formulation of the problem (2.4)-(2.10) can be deduced by density and we have

$$
\left\{\begin{array}{l}
\rho_{f} \int_{\Omega_{f}} \frac{\partial \mathbf{u}}{\partial t} \mathbf{v} d x+(A \mathbf{u}, \mathbf{v})+\rho_{f} b(\mathbf{u}, \mathbf{u}, \mathbf{v})=\int_{\Gamma_{i n}} \mathbf{h v} d \sigma+\int_{\Gamma_{w_{1}}} \mathbf{g v} d \sigma \forall \mathbf{v} \in V \\
\mathbf{u}(0)=\mathbf{u}_{0}, \quad \text { in } \Omega_{f}
\end{array}\right.
$$

\subsection{Preliminary Results}

To prove the existence of a weak solution of the problem (2.4)-(2.10) we need some preliminary results which are given in the following.

Lemma 2.1. We suppose that the function $\mu$ satisfies the following assumptions:

1. $\mu: \mathbb{R}_{+} \longrightarrow \mathbb{R}_{+}$,

2. $\mu$ is continuous,

3. $\lim _{s \rightarrow+\infty}[\mu(s)]=\mu_{0}$ with $\mu_{0}>0$,

4. $\mu$ is continuously differentiable,

5. $s\left|\mu^{\prime}(s)\right| \leq \mu(s), \forall s \in \mathbb{R}_{+}$,

6. $b=\inf _{s \in \mathbb{R}_{+}} \mu(s)>0$.

Then A, as defined in (2.11), is an hemicontinuous monotone operator, satisfying for all $(\mathbf{v}, \mathbf{w}) \in V^{2}$

$$
(A \mathbf{v}-A \mathbf{w}, \mathbf{v}-\mathbf{w}) \geq b\|\mathbf{v}-\mathbf{w}\|^{2} .
$$

Proof. The proof can be found in [15] and [14].

Lemma 2.2. Let $\Omega_{f}$ be an open bounded domain of $\mathbb{R}^{2}$ with Lipschitz boundary $\partial \Omega_{f}$. Then $C^{\infty}\left(\overline{\Omega_{f}}\right)$ is dense in $H_{1}\left(\Omega_{f}\right)$ and the application

$$
\begin{gathered}
\gamma_{0}: C^{\infty}\left(\overline{\Omega_{f}}\right) \longrightarrow L^{2}\left(\partial \Omega_{f}\right) \\
\mathbf{v} \longmapsto \gamma_{0}(\mathbf{v})=\left.\mathbf{v}\right|_{\partial \Omega_{f}}
\end{gathered}
$$

extends to a continuous linear operator $\gamma_{0}$ from $H_{1}\left(\Omega_{f}\right)$ to $L^{2}\left(\partial \Omega_{f}\right)$ called trace operator.

Proof. For the proof of the lemma, the reader can refer to [12].

Lemma 2.3. For all $\mathbf{v}, \mathbf{w} \in V$, the linear form $b$ as defined in (2.12) verifies:

1. $b(\mathbf{v}, \mathbf{v}, \mathbf{v})=b(\mathbf{v}, \mathbf{w}, \mathbf{w})=0$,

2. $b(\mathbf{v}, \mathbf{v}, \mathbf{w})=-b(\mathbf{v}, \mathbf{w}, \mathbf{v})$. 
Proof. Let first $(\mathbf{v}, \mathbf{w}) \in \mathcal{V}^{2}$, we will prove that $b(\mathbf{v}, \mathbf{w}, \mathbf{w})=0$.

Since $\mathbf{v}$ is divergence free, Green formula gives

$$
b(\mathbf{v}, \mathbf{w}, \mathbf{w})=\sum_{i, j=1}^{2} \int_{\partial \Omega_{f}} \mathbf{v}_{i} \mathbf{w}_{j} \mathbf{n}_{i} \mathbf{w}_{j} d \sigma-b(\mathbf{v}, \mathbf{w}, \mathbf{w})
$$

although

$$
\sum_{i, j=1}^{2} \int_{\Gamma_{\text {in }}} \mathbf{v}_{i} \mathbf{w}_{j} \mathbf{n}_{i} \mathbf{w}_{j} d \sigma+\sum_{i, j=1}^{2} \int_{\Gamma_{\text {out }}} \mathbf{v}_{i} \mathbf{w}_{j} \mathbf{n}_{i} \mathbf{w}_{j} d \sigma=0 .
$$

From Lemma 2, (2.14) arise for all $(\mathbf{v}, \mathbf{w}) \in \mathcal{V}^{2}$ and according to the construction of $\mathcal{V}$

$$
\sum_{i, j=1}^{2} \int_{\Gamma_{w_{1}}} \mathbf{v}_{i} \mathbf{w}_{j} \mathbf{n}_{i} \mathbf{w}_{j} d \sigma=\sum_{j=1}^{2} \int_{\Gamma_{w_{1}}} \mathbf{w}_{j}^{2} \sum_{i=1}^{2} \mathbf{v}_{i} \mathbf{n}_{i} d \sigma=0
$$

hence

$$
b(\mathbf{v}, \mathbf{w}, \mathbf{w})=0 \forall \mathbf{v} \in \mathcal{V}, \forall \mathbf{w} \in \mathcal{V} .
$$

And now, using the density of $\mathcal{V}$ into $V$ we deduce that the above equality holds for all $(\mathbf{v}, \mathbf{w}) \in V^{2}$, in particular, if $\mathbf{v}=\mathbf{w}$ we obtain $b(\mathbf{v}, \mathbf{v}, \mathbf{v})=0, \forall \mathbf{v} \in V$. The same technique is used to demonstrate that $b(\mathbf{v}, \mathbf{v}, \mathbf{w})=-b(\mathbf{v}, \mathbf{w}, \mathbf{v}) \forall(\mathbf{v}, \mathbf{w}) \in V^{2}$.

Lemma 2.4. Let $\left\{\mathbf{u}_{n}\right\}_{n \in \mathbb{N}}$ be a sequence in $L^{2}\left(0, T ;\left(L^{2}\left(\Omega_{f}\right)\right)^{2}\right)$ such that $\mathbf{u}_{n} \rightarrow \mathbf{u}$ in $L^{2}\left(0, T ;\left(L^{2}\left(\Omega_{f}\right)\right)^{2}\right)$ and $\mathbf{u}_{n} \rightarrow \mathbf{u}$ in $L^{2}(0, T ; V)$ then for all $\mathbf{w} \in \mathcal{C}^{1}(\bar{Q})$

$$
\int_{0}^{T} b\left(\mathbf{u}_{n}(t), \mathbf{u}_{n}(t), \mathbf{w}_{j}\right) d t \rightarrow \int_{0}^{T} b\left(\mathbf{u}(t), \mathbf{u}(t), \mathbf{w}_{j}\right) d t
$$

with $Q=\Omega \times[0, T]$.

Proof. The proof is based on the second part of the previous lemma, then we apply the same technique as in $[8]$.

Lemma 2.5. We assume that $\gamma \in L^{2}\left(0, T ;\left(L^{2}(\Gamma)\right)^{2}\right)$ such that $\Gamma$ is a part of $\partial \Omega_{f}$ and we consider the following application:

$$
\begin{aligned}
\mathcal{T}_{\gamma}: & L^{2}(0, T ; V) \longrightarrow \mathbb{R} \\
& \mathbf{w} \longmapsto \int_{0}^{T} \int_{\Gamma} \gamma(x, t) \mathbf{w}(x, t) d x d t
\end{aligned}
$$

then $\mathcal{T}_{\gamma}$ is well-defined, linear and continuous.

Proof. It is clear that the application is linear and continuous. Indeed,

$$
\begin{aligned}
\left|\mathcal{T}_{\gamma}(\mathbf{w})\right| & =\left|\sum_{i=1}^{2} \int_{0}^{T} \int_{\Gamma} \gamma_{i}(x, t) \mathbf{w}_{i}(x, t) d x d t\right| \leq \sum_{i=1}^{2} \int_{0}^{T} \int_{\partial \Omega_{f}}\left|\gamma_{i}(x, t) \mathbf{w}_{i}(x, t)\right| d x d t \\
& \leq \sum_{i=1}^{2}\left(\int_{0}^{T}\left(\int_{\partial \Omega_{f}}\left(\gamma_{i}(x, t)\right)^{2} d x\right) d t\right)^{\frac{1}{2}}\left(\int_{0}^{T}\left(\int_{\partial \Omega_{f}}\left(\mathbf{w}_{i}(x, t)\right)^{2} d x\right) d t\right)^{\frac{1}{2}}
\end{aligned}
$$




$$
\begin{gathered}
\leq\|\gamma\|_{L^{2}\left(0, T ;\left(L^{2}\left(\partial \Omega_{f}\right)\right)^{2}\right)}\left(\sum_{i=1}^{2}\left(\int_{0}^{T}\left(\int_{\partial \Omega_{f}}\left(\mathbf{w}_{i}(x, t)\right)^{2} d x\right) d t\right)\right)^{\frac{1}{2}} \\
\leq K\|\gamma\|_{L^{2}\left(0, T ;\left(L^{2}\left(\partial \Omega_{f}\right)\right)^{2}\right)}\|\mathbf{w}\|_{L^{2}(0, T ; V)} .
\end{gathered}
$$

Theorem 2.6. (Compactness theorem [8])

Let $B_{0}$ and $B$ be two Banach spaces and $B_{1}$ is a Hilbert space such that $B_{0} \hookrightarrow B \hookrightarrow B_{1}$ with continuous imbeddings and the imbedding $B_{0} \hookrightarrow B$ is compact. Let then

$$
Z=\left\{\mathbf{v} \mid \mathbf{v} \in L^{p_{0}}\left(0, T ; B_{0}\right), \quad \frac{\partial \mathbf{v}}{\partial t} \in L^{1}\left(0, T ; B_{1}\right)\right\} .
$$

Then, under the previous hypotheses, the imbedding of $Z$ in $L^{p_{0}}(0, T ; B)$ is compact for any finite number $p_{0}>1$.

\subsection{Existence Result}

We consider, at first on $[0, T] \times \Omega_{f}$, the following problem

$$
\begin{gathered}
\rho_{f} \frac{\partial \mathbf{u}}{\partial t}+\rho_{f}((\mathbf{u}-w) \cdot \nabla) \mathbf{u}-\nabla \cdot(2 \mu(\mathbf{s}(\mathbf{u})) \mathbf{D u})+\nabla p=0, \text { in } \Omega_{f} \times(0, T), \\
\nabla \cdot \mathbf{u}=0, \text { in } \Omega_{f} \times(0, T) . \\
2 \mu(\mathbf{s}(\mathbf{u})) \mathbf{D u} \cdot \mathbf{n}-p \mathbf{n}=\mathbf{h}, \text { on } \Gamma_{\text {in }} \times(0, T), \\
2 \mu(\mathbf{s}(\mathbf{u})) \mathbf{D u} \cdot \mathbf{n}-p \mathbf{n}=0, \text { on } \Gamma_{\text {out }} \times(0, T), \\
\mathbf{u} \cdot \mathbf{n}=0 \text { and } 2 \mu(\mathbf{s}(\mathbf{u})) \mathbf{D u} \cdot \mathbf{n}-p \mathbf{n}=\mathbf{g}, \text { on } \Gamma_{w_{1}} \times(0, T), \\
\mathbf{u}=0, \text { on } \Gamma_{w_{2}} \times(0, T), \\
\mathbf{u}=\mathbf{u}_{0}, \text { for } t=0 \text { in } \Omega_{f},
\end{gathered}
$$

where $\mathbf{n}$ is the outward normal vector, $\rho_{f}, \mu$ and $w$ are given. $\mathbf{u}$ and $p$ are unknown functions.

Notice that the problem (2.4)-(2.10) is a special case of the previous one when we take $w=0$. We will prove in this subsection an existence result for a weak solution of the problem (2.15)-(2.21).

Theorem 2.7. Let $\mathbf{u}_{0} \in\left(L^{2}\left(\Omega_{f}\right)\right)^{2}, \mathbf{h} \in L^{2}\left(0, T ;\left(L^{2}\left(\Gamma_{i n}\right)\right)^{2}\right), \mathbf{g} \in L^{2}\left(0, T ;\left(L^{2}\left(\Gamma_{w_{1}}\right)\right)^{2}\right)$, $w \in L^{2}(0, T, V)$ and $\mu$ satisfying the assumptions of Lemma 1. Then the problem (2.15)-(2.21) admits at least one weak solution

$$
\mathbf{u} \in L^{2}(0, T ; V) \cap L^{\infty}\left(0, T ;\left(L^{2}\left(\Omega_{f}\right)\right)^{2}\right)
$$

such that

$$
\mathbf{u}^{\prime} \in L^{1}\left(0, T ; V^{\prime}\right) \quad\left(\mathbf{u}^{\prime}=\frac{d \mathbf{u}}{d t} \text { and } V^{\prime} \text { is the dual space of } V\right)
$$


Proof. A weak formulation of the problem (2.15)-(2.21) can be deduced by density and we have

$$
\left\{\begin{array}{l}
\rho_{f} \int_{\Omega_{f}} \frac{\partial \mathbf{u}}{\partial t} \mathbf{v} d x+(A \mathbf{u}, \mathbf{v})+\rho_{f} b(\mathbf{u}, \mathbf{u}, \mathbf{v})-\rho_{f} b(w, \mathbf{u}, \mathbf{v})= \\
\int_{\Gamma_{i n}} \mathbf{h v} d \sigma+\int_{\Gamma_{w_{1}}} \mathbf{g v} d \sigma \forall \mathbf{v} \in V \\
\mathbf{u}(0)=\mathbf{u}_{0}, \quad \text { in } \Omega_{f} .
\end{array}\right.
$$

The classical tool that will be introduced here is known as the Faedo Galerkin method where we define a series of differential equations system for which the existence of a discrete solution follows from Cauchy theorem. Different a priori estimates are then derived and provide uniform bounds on the sequence of solutions. This is the corner stone of the proof, as it allows us to state that there exists a subsequence of discrete solutions that converges to a solution of (2.22) when the dimension of the finite dimensional subspaces goes to infinity.

\section{Approximated solutions}

We consider the spectral problem

$$
((\mathbf{w}, \mathbf{v}))=\lambda(\mathbf{w}, \mathbf{v}) \forall \mathbf{v} \in V
$$

Since $V$ is closed subspace of $\left(H^{1}\left(\Omega_{f}\right)\right)^{2}$ and the injection of $\left(H^{1}\left(\Omega_{f}\right)\right)^{2}$ in $\left(L^{2}\left(\Omega_{f}\right)\right)^{2}$ is compact, the problem (2.23) admits a sequence of eigenvalues $\lambda_{j}$ corresponding to eigenvectors $\mathbf{w}_{j}$ such that

$$
\left(\left(\mathbf{w}_{j}, \mathbf{v}\right)\right)=\lambda_{j}\left(\mathbf{w}_{j}, \mathbf{v}\right) \forall \mathbf{v} \in V
$$

and $\left\{\mathbf{w}_{j}\right\}_{j \in \mathbb{N}}$ is orthonormal in $\left(L^{2}\left(\Omega_{f}\right)\right)^{2}$ and orthogonal in $V$.

Let $N \in \mathbb{N}^{*}$, if we denote by $\left[\mathbf{w}_{1}, \ldots \mathbf{w}_{N}\right]$ the subspace spaned by $\mathbf{w}_{1}, \ldots \mathbf{w}_{N}$ then

$$
V={\overline{\left[\mathbf{w}_{1} \cdots \mathbf{w}_{N}\right]}}^{V} .
$$

We denote by $\mathbf{u}_{N}(t)$ the approximate solution of (2.13) defined by

$$
\left\{\begin{array}{l}
\mathbf{u}_{N}(x, t)=\mathbf{u}_{N}(t)(x) \in\left[\mathbf{w}_{1}, \ldots \mathbf{w}_{N}\right] \\
\mathbf{u}_{N}(x, t)=\sum_{j=1}^{N} C_{j}^{N}(t) \mathbf{w}_{j}(x) \\
\mathbf{u}_{N}(\cdot, 0)=\mathbf{u}_{0 N}(\cdot) \in\left[\mathbf{w}_{1}, \ldots \mathbf{w}_{N}\right]
\end{array}\right.
$$

where $\mathbf{u}_{0 N}=\sum_{i=1}^{N}\left(\mathbf{u}_{0}, \mathbf{w}_{i}\right) \mathbf{w}_{i}$ and $\mathbf{u}_{0 N} \stackrel{N \rightarrow \infty}{\rightarrow} \mathbf{u}_{0}$ in $\left(L^{2}\left(\Omega_{f}\right)\right)^{2}$. We have then

$$
\left\{\begin{array}{l}
\rho_{f}\left(\mathbf{u}_{N}^{\prime}(t), \mathbf{w}_{j}\right)+\left(A \mathbf{u}_{N}(t), \mathbf{w}_{j}\right) \\
+\rho_{f} b\left(\mathbf{u}_{N}(t), \mathbf{u}_{N}(t), \mathbf{w}_{j}\right)-\rho_{f} b\left(w(t), \mathbf{u}_{N}(t), \mathbf{w}_{j}\right)=\int_{\Gamma_{i n}} \mathbf{h} \mathbf{w}_{j} d \sigma+\int_{\Gamma_{w_{1}}} \mathbf{g w}_{j} d \sigma, \\
\mathbf{u}_{N}(\cdot, t)=\mathbf{u}_{0} N(\cdot) \in\left[\mathbf{w}_{1}, \ldots \mathbf{w}_{N}\right] \quad 1 \leq j \leq N .
\end{array}\right.
$$

this set of equations yields a system of nonlinear differential equations in the components $C_{j}^{N}(t)$, which defines $\mathbf{u}_{N}(t)$ a solution of $(2.24)$ on $\left[0, T_{N}\right] \subset[0, T]$.

We will see in the next point that we can take $T_{N}=T$ for all $N \in \mathbb{N}$.

Derivation of a priori estimates

Multiplying (2.24) by $C_{j}^{N}(t)$ and adding the obtained equations we deduce, thanks to Lemma 3 , that:

$$
\rho_{f} \frac{1}{2} \frac{d}{d t}\left|\mathbf{u}_{N}(t)\right|^{2}+\left(A \mathbf{u}_{N}(t), \mathbf{u}_{N}(t)\right)=\int_{\Gamma_{i n}} \mathbf{h} \mathbf{u}_{N}(t) d \sigma+\int_{\Gamma_{w_{1}}} \mathbf{g} \mathbf{u}_{N}(t) d \sigma,
$$


from the assumptions of lemma 1, we have

$$
\int_{\Omega_{f}} \mu\left(\mathbf{s}\left(\mathbf{u}_{N}(t)\right)\right) \mathbf{s}^{2}\left(\mathbf{u}_{N}(t)\right) d x \geq b \int_{\Omega_{f}} \mathbf{s}^{2}\left(\mathbf{u}_{N}(t)\right) d x
$$

and (2.25) implies

$$
\rho_{f} \frac{d}{d t}\left|\mathbf{u}_{N}(t)\right|^{2}+2 b \int_{\Omega_{f}} \mathbf{s}^{2}\left(\mathbf{u}_{N}(t)\right) d x \leq \int_{\Gamma_{i n}} \mathbf{h} \mathbf{u}_{N}(t) d \sigma+\int_{\Gamma_{w_{1}}} \mathbf{g u}_{N}(t) d \sigma .
$$

Otherwise, using Hölder inequality, we have

$$
\int_{\Gamma_{i n}} \mathbf{h} \mathbf{u}_{N}(t) d \sigma \leq|\mathbf{h}|_{L^{2}\left(\Gamma_{i n}\right)}\left|\mathbf{u}_{N}(t)\right|_{L^{2}\left(\Gamma_{i n}\right)}
$$

and by trace theorem

$$
|\mathbf{h}|_{L^{2}\left(\Gamma_{i n}\right)}\left|\mathbf{u}_{N}(t)\right|_{L^{2}\left(\Gamma_{i n}\right)} \leq K|\mathbf{h}|_{L^{2}\left(\Gamma_{i n}\right)}|| \mathbf{u}_{N}(t) \|_{H^{1}\left(\Omega_{f}\right)} .
$$

Applying Poincaré inequality we get

$$
K|\mathbf{h}|_{L^{2}\left(\Gamma_{i n}\right)}|| \mathbf{u}_{N}(t) \|_{H^{1}\left(\Omega_{f}\right)} \leq K C\left(\Omega_{f}\right)|\mathbf{h}|_{L^{2}\left(\Gamma_{i n}\right)}\left|\nabla \mathbf{u}_{N}(t)\right|_{L^{2}\left(\Omega_{f}\right)}
$$

and from Korn inequality we obtain

$$
K C\left(\Omega_{f}\right)|\mathbf{h}|_{L^{2}\left(\Gamma_{i n}\right)}\left|\nabla \mathbf{u}_{N}(t)\right|_{L^{2}\left(\Omega_{f}\right)} \leq\left. K M C\left(\Omega_{f}\right)|\mathbf{h}|_{L^{2}\left(\Gamma_{i n}\right)}|| \mathbf{D} \mathbf{u}_{N}(t)\right|_{L^{2}\left(\Omega_{f}\right)},
$$

moreover

$$
K C\left(\Omega_{f}\right)|\mathbf{h}|_{L^{2}\left(\Gamma_{i n}\right)}\left|\nabla \mathbf{u}_{N}(t)\right|_{L^{2}\left(\Omega_{f}\right)} \leq \frac{K^{2} M^{2} C^{2}\left(\Omega_{f}\right)}{2 b}|\mathbf{h}|_{L^{2}\left(\Gamma_{i n}\right)}^{2 b}+\frac{b}{2}\left\|\mathbf{D} \mathbf{u}_{N}(t)\right\|_{L^{2}\left(\Omega_{f}\right)}^{2},
$$

hence, by the same reasoning, we deduced that

$$
\int_{\Gamma_{w_{1}}} \mathbf{g u}_{N}(t) d \sigma \leq \frac{K^{2} M^{2} C^{2}\left(\Omega_{f}\right)}{2 b}|\mathbf{g}|_{L^{2}\left(\Gamma_{w_{1}}\right)}^{2}+\frac{b}{2}\left\|\mathbf{D} \mathbf{u}_{N}(t)\right\|_{L^{2}\left(\Omega_{f}\right)}^{2} .
$$

From (2.26) we have

$$
\rho_{f} \frac{d}{d t}\left|\mathbf{u}_{N}(t)\right|^{2}+\left.b|| \mathbf{D} \mathbf{u}_{N}(t)\right|_{L^{2}\left(\Omega_{f}\right)} ^{2} \leq C\left(|\mathbf{h}(t)|_{L^{2}\left(\Gamma_{i n}\right)}^{2}+|\mathbf{g}(t)|_{L^{2}\left(\Gamma_{w_{1}}\right)}^{2}\right),
$$

it results from integrating $(2.27)$ over $[0, T]$

$$
\rho_{f}\left|\mathbf{u}_{N}(t)\right|^{2}+b \int_{0}^{T}|| \mathbf{D} \mathbf{u}_{N}(t) \|_{L^{2}\left(\Omega_{f}\right)}^{2} d t \leq C\left(\int_{0}^{T}|\mathbf{h}(t)|_{L^{2}\left(\Gamma_{i n}\right)}^{2} d t+\int_{0}^{T}|\mathbf{g}(t)|_{L^{2}\left(\Gamma_{w_{1}}\right)}^{2} d t\right)+\rho_{f}\left|\mathbf{u}_{N 0}\right|^{2},
$$

thus, there are two constants $C_{1}>0$ and $C_{2}>0$ such that

$$
\left|\mathbf{u}_{N}(t)\right| \leq C_{1} \text { and } \int_{0}^{T}\left\|\mathbf{D} \mathbf{u}_{N}(t)\right\|_{L^{2}\left(\Omega_{f}\right)}^{2} d t \leq C_{2} \forall t \in\left[0, T_{N}\right]
$$

We first deduce that $T_{N}=T$ and moreover

$$
\mathbf{u}_{N} \text { is a bounded sequence in } L^{2}(0, T ; V) \cap L^{\infty}\left(0, T ;\left(L^{2}\left(\Omega_{f}\right)\right)^{2}\right) \text {. }
$$

We will also prove that for $N \in \mathbb{N}$ : 
$\mathbf{u}_{N}^{\prime}$ is a bounded sequence in $L^{1}\left(0, T ; V^{\prime}\right)$.

To do this, we first establish that:

$$
\begin{aligned}
& A \mathbf{u}_{N} \text { is a bounded sequence in } L^{2}\left(0, T ; V^{\prime}\right), \\
& B \mathbf{u}_{N} \text { is a bounded sequence in } L^{1}\left(0, T ; V^{\prime}\right)
\end{aligned}
$$

and

$C \mathbf{u}_{N}$ is a bounded sequence in $L^{1}\left(0, T ; V^{\prime}\right)$

where the operators $A, B$ and $C$ are defined, for all $(u, v, w) \in V^{3}$, as follows

$$
\begin{gathered}
(A \mathbf{u}, \mathbf{v})=\int_{\Omega_{f}}(2 \mu(s(\mathbf{u})) \mathbf{D u}, \mathbf{D v}), \\
(B \mathbf{u}, \mathbf{v})=b(\mathbf{u}, \mathbf{u}, \mathbf{v})
\end{gathered}
$$

and

$$
(C \mathbf{u}, \mathbf{v})=b(w, \mathbf{u}, \mathbf{v})
$$

Indeed, for all $\mathbf{v} \in V, \sup _{s \in \mathbb{R}_{+}} \mu(s)=a$ and from the assumptions of lemma 1, we have

$$
\left(A \mathbf{u}_{N}(t), \mathbf{v}\right)=\int_{\Omega_{f}}\left(2 \mu\left(s\left(\mathbf{u}_{N}(t)\right)\right) \mathbf{D} \mathbf{u}_{N}(t), \mathbf{D v}\right) \leq 2 a\left\|\mathbf{u}_{N}(t)\right\|\|\mathbf{v}\|
$$

whence by duality

$$
\left\|A \mathbf{u}_{N}(t)\right\|_{V^{\prime}} \leq 2 a\left\|\mathbf{u}_{N}(t)\right\|
$$

and from (2.28) we deduct

$$
\int_{0}^{T}\left\|A \mathbf{u}_{N}(t)\right\|_{V^{\prime}}^{2} d t \leq 4 a^{2} \int_{0}^{T}\left\|\mathbf{u}_{N}(t)\right\|^{2} d t \leq C_{A} .
$$

On the other hand, from the definition of $b\left(\mathbf{u}_{N}(t), \mathbf{u}_{N}(t), \mathbf{v}\right)$ and applying Hölder we have

$$
b\left(\mathbf{u}_{N}(t), \mathbf{u}_{N}(t), \mathbf{v}\right)=\sum_{i, j=1}^{2} \int_{\Omega_{f}} \mathbf{u}_{N, i} \frac{\partial \mathbf{u}_{N, j}}{\partial x_{i}} \mathbf{v}_{j} d x \leq \sum_{i, j=1}^{2}\left(\int_{\Omega_{f}}\left(\frac{\partial \mathbf{u}_{N, j}}{\partial x_{i}}\right)^{2} d x\right)^{\frac{1}{2}}\left(\int_{\Omega_{f}} \mathbf{u}_{N, i}^{2} \mathbf{v}_{j}^{2} d x\right)^{\frac{1}{2}} .
$$

Hölder is applied a second time, but on the second integral of the second term of the inequality

$$
\sum_{i, j=1}^{2}\left(\int_{\Omega_{f}}\left(\frac{\partial \mathbf{u}_{N, j}}{\partial x_{i}}\right)^{2} d x\right)^{\frac{1}{2}}\left(\int_{\Omega_{f}} \mathbf{u}_{N, i}^{2} \mathbf{v}_{j}^{2} d x\right)^{\frac{1}{2}} \leq \sum_{i, j=1}^{2}\left(\int_{\Omega_{f}}\left(\frac{\partial \mathbf{u}_{N, j}}{\partial x_{i}}\right)^{2} d x\right)^{\frac{1}{2}}\left\|\mathbf{u}_{N, i}\right\|_{L^{4}\left(\Omega_{f}\right)}\left\|\mathbf{v}_{j}\right\|_{L^{4}\left(\Omega_{f}\right)},
$$

however the continuous imbeeding theorem allows us to write

$$
\sum_{i, j=1}^{2}\left(\int_{\Omega_{f}}\left(\frac{\partial \mathbf{u}_{N, j}}{\partial x_{i}}\right)^{2} d x\right)^{\frac{1}{2}}\left\|\mathbf{u}_{N, i}\right\|_{L^{4}\left(\Omega_{f}\right)}\left\|\mathbf{v}_{j}\right\|_{L^{4}\left(\Omega_{f}\right)} \leq C_{b}^{2} \sum_{i, j=1}^{2}\left(\int_{\Omega_{f}}\left(\frac{\partial \mathbf{u}_{N, j}}{\partial x_{i}}\right)^{2} d x\right)^{\frac{1}{2}}\left\|\mathbf{u}_{N, i}\right\|_{H^{1}\left(\Omega_{f}\right)}\left\|\mathbf{v}_{j}\right\|_{H^{1}\left(\Omega_{f}\right)},
$$

or else

$$
C_{b}^{2} \sum_{j=1}^{2}\left\|\mathbf{v}_{j}\right\|_{H^{1}\left(\Omega_{f}\right)}\left(\sum_{i=1}^{2}\left(\int_{\Omega_{f}}\left(\frac{\partial \mathbf{u}_{N, j}}{\partial x_{i}}\right)^{2} d x\right)^{\frac{1}{2}}\left\|\mathbf{u}_{N, i}\right\|_{H^{1}\left(\Omega_{f}\right)}\right) \leq C_{b}^{2}\left\|\mathbf{u}_{N}(t)\right\|_{\left(H^{1}\left(\Omega_{f}\right)\right)^{2}}^{2}\|\mathbf{v}\|_{\left(H^{1}\left(\Omega_{f}\right)\right)^{2}}
$$


therefore

$$
b\left(\mathbf{u}_{N}(t), \mathbf{u}_{N}(t), \mathbf{v}\right)=\left(B \mathbf{u}_{N}(t), \mathbf{v}\right) \leq K_{b}\left\|\mathbf{u}_{N}(t)\right\|_{\left(H^{1}\left(\Omega_{f}\right)\right)^{2}}^{2}\|\mathbf{v}\|_{\left(H^{1}\left(\Omega_{f}\right)\right)^{2}},
$$

whence by duality

$$
\left\|B \mathbf{u}_{N}(t)\right\|_{V^{\prime}} \leq K_{b}\left\|\mathbf{u}_{N}(t)\right\|_{\left(H^{1}\left(\Omega_{f}\right)\right)^{2}}^{2} .
$$

Finally, in the same way, we can deduce as bellow that

$$
\left\|C \mathbf{u}_{N}(t)\right\|_{V^{\prime}} \leq K_{b}\|w(t)\|_{\left(H^{1}\left(\Omega_{f}\right)\right)^{2}}\left\|\mathbf{u}_{N}(t)\right\|_{\left(H^{1}\left(\Omega_{f}\right)\right)^{2}},
$$

then

$A \mathbf{u}_{N}$ is a bounded sequence in $L^{2}\left(0, T ; V^{\prime}\right)$

and $B \mathbf{u}_{N}$ and $C \mathbf{u}_{N}$ are bounded sequences in $L^{1}\left(0, T ; V^{\prime}\right)$.

Furthermore, let first $\mathbf{v} \in\left[\mathbf{w}_{1} \cdots \mathbf{w}_{N}\right]$, then $\mathbf{v}(x)=\sum_{j=1}^{N} \alpha_{j} \mathbf{w}_{j}(x)$ for all $x \in \Omega_{f} . \mathbf{v}$ is then substituted in (2.13) by this last expression and it results that

$$
\begin{aligned}
\rho_{f}\left(\mathbf{u}_{N}^{\prime}(t), \mathbf{v}\right) & =\rho_{f} \sum_{j=1}^{N} \alpha_{j}\left(\mathbf{u}_{N}^{\prime}(t), \mathbf{w}_{j}\right) \\
& =-\sum_{j=1}^{N} \alpha_{j}\left(A \mathbf{u}_{N}(t), \mathbf{w}_{j}\right)-\rho_{f} \sum_{j=1}^{N} \alpha_{j} b\left(\mathbf{u}_{N}(t), \mathbf{u}_{N}(t), \mathbf{w}_{j}\right) \\
& +\rho_{f} \sum_{j=1}^{N} \alpha_{j} b\left(w(t), \mathbf{u}_{N}(t), \mathbf{w}_{j}\right)+\sum_{j=1}^{N} \alpha_{j} \int_{\Gamma_{i n}} \mathbf{h}(t) \mathbf{w}_{j} d \sigma \\
& +\sum_{j=1}^{N} \alpha_{j} \int_{\Gamma_{w_{1}}} \mathbf{g}(t) \mathbf{w}_{j} d \sigma, \quad t \in[0, T] .
\end{aligned}
$$

Thus, according to (2.29) for $\mathbf{w}_{j}$, we deduct from (2.30)

$$
\rho_{f}\left(\mathbf{u}_{N}^{\prime}(t), \mathbf{v}\right) \leq M\left(\left\|\mathbf{u}_{N}(t)\right\|+\left\|\mathbf{u}_{N}(t)\right\|^{2}+\left\|\left.\mathbf{u}_{N}(t)|\||| w(t)\|+| \mathbf{h}\right|_{L^{2}\left(\Gamma_{i n}\right)}+|\mathbf{g}|_{L^{2}\left(\Gamma_{w_{1}}\right)}\right)\|\mathbf{v}\| .\right.
$$

Let now $\mathbf{v} \in V$. As already mentioned the "special basis" formed by the eigenvectors $\left\{\mathbf{w}_{1} \cdots \mathbf{w}_{N}\right\}$ generates a subspace which is dense in $V$, then there exists $\left(\mathbf{v}_{p}\right)_{p} \in\left[\mathbf{w}_{1}, \ldots \mathbf{w}_{n}\right]$ such that $\mathbf{v}_{p} \longrightarrow \mathbf{v}$ in $\left(H^{1}\left(\Omega_{f}\right)\right)^{2}$. By duality, we obtain

$$
\left\|\mathbf{u}_{N}^{\prime}(t)\right\|_{V^{\prime}} \leq M\left(\left\|\mathbf{u}_{N}(t)\right\|+\left\|\mathbf{u}_{N}(t)\right\|^{2}+\left\|\mathbf{u}_{N}(t)\right\|\left\|\left.|w(t) \|+| \mathbf{h}\right|_{L^{2}\left(\partial \Omega_{f}\right)}+|\mathbf{g}|_{L^{2}\left(\partial \Omega_{f}\right)}\right) .\right.
$$

We, thus, demonstrate that

$$
\mathbf{u}_{N}^{\prime} \text { is a bounded sequence in } L^{1}\left(0, T ; V^{\prime}\right) \text {. }
$$

Passing to the limit

We now use this compactness theorem in the following situation: $p_{0}=2, p_{1}=1, B_{0}=V, B_{1}=V^{\prime}$, and we choose $B=\left(L^{2}\left(\partial \Omega_{f}\right)\right)^{2}$ and $Z=\left\{\mathbf{v} \mid \mathbf{v} \in L^{2}(0, T ; V), \quad \frac{\partial \mathbf{v}}{\partial t} \in L^{1}\left(0, T ; V^{\prime}\right)\right\}$.

Thanks to (2.28), (2.31) and the compactness theorem we deduce that we can extract a subsequence $\left\{\mathbf{u}_{m}\right\}_{m \in \mathbb{N}}$ such that

$$
\mathbf{u}_{m} \rightarrow \mathbf{u} \quad \text { in } L^{2}(0, T ; V),
$$

$\mathbf{u}_{m} \rightarrow \mathbf{u} \quad$ weak star in $L^{\infty}\left(0, T ;\left(L^{2}\left(\partial \Omega_{f}\right)\right)^{2}\right)$, 


$$
\mathbf{u}_{m} \rightarrow \mathbf{u} \text { in } L^{2}\left(0, T ;\left(L^{2}\left(\partial \Omega_{f}\right)\right)^{2}\right) \text { and a.e in } \Omega_{f} \times[0, T] .
$$

Moreover, $\mathbf{u}_{m}$ verifies (2.24) for all $m \in \mathbb{N}$.

Furthermore by (2.29)

$$
A \mathbf{u}_{m} \rightarrow \chi \text { in } L^{2}\left(0, T ; V^{\prime}\right) .
$$

Indeed, let $\psi$ be a differential continuous function in $[0, T]$ such that $\psi(T)=0$.

Multiplying the equation (2.24) by the function $\psi(T)$ and integrating by parts, it results:

$$
\begin{aligned}
& -\rho_{f} \int_{0}^{T}\left(\mathbf{u}_{m}(t), \psi^{\prime}(t) \mathbf{w}_{j}\right) d t+\int_{0}^{T}\left(A \mathbf{u}_{m}(t), \mathbf{w}_{j} \psi(t)\right) d t \\
& +\rho_{f} \int_{0}^{T} b\left(\mathbf{u}_{m}(t), \mathbf{u}_{m}(t), \mathbf{w}_{j} \psi(t)\right) d t-\rho_{f} \int_{0}^{T} b\left(w(t), \mathbf{u}_{m}(t), \mathbf{w}_{j} \psi(t)\right) d t \\
& =\rho_{f}\left(\mathbf{u}_{0 m}, \mathbf{w}_{j}\right) \psi(0)+\int_{0}^{T} \int_{\Gamma_{i n}} h \mathbf{w}_{j} \psi(t) d \sigma d t+\int_{0}^{T} \int_{\Gamma_{w_{1}}} g \mathbf{w}_{j} \psi(t) d \sigma d t .
\end{aligned}
$$

Passing to the limit in (2.32) and using Lemma 4, we obtain

$$
\begin{aligned}
& -\rho_{f} \int_{0}^{T}\left(\mathbf{u}(t), \psi^{\prime}(t) \mathbf{w}_{j}\right) d t+\int_{0}^{T}\left(\chi(t), \mathbf{w}_{j} \psi(t)\right) d t \\
& +\rho_{f} \int_{0}^{T} b\left(\mathbf{u}(t), \mathbf{u}(t), \mathbf{w}_{j} \psi(t)\right) d t-\rho_{f} \int_{0}^{T} b\left(w(t), \mathbf{u}(t), \mathbf{w}_{j} \psi(t)\right) d t \\
& =\rho_{f}\left(\mathbf{u}_{0}, \mathbf{w}_{j}\right) \psi(0)+\int_{0}^{T} \int_{\Gamma_{i n}} h \mathbf{w}_{j} \psi(t) d \sigma d t+\int_{0}^{T} \int_{\Gamma_{w_{1}}} g \mathbf{w}_{j} \psi(t) d \sigma d t,
\end{aligned}
$$

for all $\mathbf{w}_{j}$.

By density, (2.33) holds for all $\mathbf{v} \in V$.

Therefore (2.33) is particularly true for all $\mathbf{v} \in V$ and $\psi \in \mathcal{D}(0, T)$. After integration by parts in the first integral we can deduce that:

$$
\rho_{f}\left(\mathbf{u}^{\prime}(t), \mathbf{v}\right)+(\chi(t), \mathbf{v})+\rho_{f} b(\mathbf{u}(t), \mathbf{u}(t), \mathbf{v})=\int_{\Gamma_{i n}} \mathbf{h v} d \sigma+\int_{\Gamma_{w_{1}}} \mathbf{g v} d \sigma, \quad \forall \mathbf{v} \in V,
$$

in the distributions sens. Moreover, by multiplying (2.34) by $\psi$, a continuously differentiable function such that $\psi(t)=0$, and integrating by parts, we get:

$$
\begin{aligned}
& -\rho_{f} \int_{0}^{T}\left(\mathbf{u}(t), \psi^{\prime}(t) \mathbf{v}\right) d t+\int_{0}^{T}(\chi(t), \mathbf{v} \psi(t)) d t \\
& \quad+\rho_{f} \int_{0}^{T} b(\mathbf{u}(t), \mathbf{u}(t), \mathbf{v} \psi(t)) d t-\rho_{f} \int_{0}^{T} b(w(t), \mathbf{u}(t), \mathbf{v} \psi(t)) d t \\
& \quad=\rho_{f}(\mathbf{u}(0), \mathbf{v}) \psi(0)+\int_{0}^{T} \int_{\Gamma_{i n}} h \mathbf{v} \psi(t) d \sigma d t+\int_{0}^{T} \int_{\Gamma_{w_{1}}} g \mathbf{v} \psi(t) d \sigma d t .
\end{aligned}
$$

Comparing with (2.33) written for $\mathbf{v} \in V$ we have:

$$
\left(\mathbf{u}(0)-\mathbf{u}_{0}, \mathbf{v}\right) \psi(0)=0 .
$$

We can choose $\psi$ such that $\psi(0)=1$, therefore

$$
\left(\mathbf{u}(0)-\mathbf{u}_{0}, \mathbf{v}\right)=0, \quad \forall \mathbf{v} \in V .
$$

We will now prove that

$$
(\chi(t), \mathbf{v})=(A \mathbf{u}(t), \mathbf{v}), \quad \forall \mathbf{v} \in V, \quad \forall t \in[0, T] .
$$

To do this, we will use a method of monotony and Lemma 5.

Let $m \in \mathbb{N}$, we set:

$$
X_{m}=\int_{0}^{T}\left(A \mathbf{u}_{m}(t)-A \mathbf{v}, \mathbf{u}_{m}(t)-\mathbf{v}\right) d t, \quad \forall \mathbf{v} \in V
$$


Since $A$ is monotone, we deduce that

$$
X_{m} \geq 0, \quad \forall m \in \mathbb{N}
$$

yet

$$
\int_{0}^{T}\left(A \mathbf{u}_{m}(t), \mathbf{u}_{m}(t)\right) d t=\int_{0}^{T} \int_{\Gamma_{i n}} \mathbf{h} \mathbf{u}_{m}(t) d \sigma d t+\int_{0}^{T} \int_{\Gamma_{w_{1}}} \mathbf{g} \mathbf{u}_{m}(t) d \sigma d t+\frac{1}{2}\left|\mathbf{u}_{0 m}\right|^{2}-\frac{1}{2}\left|\mathbf{u}_{m}(T)\right|^{2}
$$

and then

$$
\begin{gathered}
X_{m}=\int_{0}^{T} \int_{\Gamma_{i n}} \mathbf{h} \mathbf{u}_{m}(t) d \sigma d t+\int_{0}^{T} \int_{\Gamma_{w_{1}}} \mathbf{g} \mathbf{u}_{m}(t) d \sigma d t+\frac{1}{2}\left|\mathbf{u}_{0 m}\right|^{2} \\
-\frac{1}{2}\left|\mathbf{u}_{m}(T)\right|^{2}-\int_{0}^{T}\left(A \mathbf{u}_{m}(t), \mathbf{v}\right) d t-\int_{0}^{T}\left(A \mathbf{v}, \mathbf{u}_{m}(t)-v\right) d t
\end{gathered}
$$

since $\mathbf{u}_{m}(t) \rightarrow \mathbf{u}(t)$ in $L^{2}(\Omega)$ and from the Lemma 5 it follows:

$$
\liminf \left|\mathbf{u}_{m}(t)\right| \geq|\mathbf{u}(t)|
$$

whence

$$
\begin{gathered}
0 \leq \limsup X_{m} \leq \int_{0}^{T}<\mathcal{T}_{\mathbf{h}}, \mathbf{u}(t)>d t+\int_{0}^{T}<\mathcal{T}_{\mathbf{g}}, \mathbf{u}(t)>d t+\frac{1}{2}\left|\mathbf{u}_{0}\right|^{2} \\
-\frac{1}{2}|\mathbf{u}(T)|^{2}-\int_{0}^{T}(\chi(t), \mathbf{v}) d t-\int_{0}^{T}(A \mathbf{v}, \mathbf{u}(t)-v) d t
\end{gathered}
$$

but

$$
\int_{0}^{T}<\mathcal{T}_{\mathbf{h}}, \mathbf{u}(t)>d t+\int_{0}^{T}<\mathcal{T}_{\mathbf{g}}, \mathbf{u}(t)>d t+\frac{1}{2}\left|\mathbf{u}_{0}\right|^{2}-\frac{1}{2}|\mathbf{u}(T)|^{2}=\int_{0}^{T}(\chi(t), \mathbf{u}(t)) d t
$$

(if we take $\mathbf{v}=\mathbf{u}(t)$ in (2.34) and integrating over $[0, T]$ ). Then

$$
\int_{0}^{T}(\chi(t)-A \mathbf{v}, \mathbf{u}(t)-\mathbf{v}) d t \geq 0, \quad \forall \mathbf{v} \in V .
$$

Furthermore, let $\lambda>0$ and $\mathbf{w} \in V$. We set $\mathbf{v}=\mathbf{u}(t)-\lambda \mathbf{w}$ then $\mathbf{v} \in V$ and we have:

$$
\lambda \int_{0}^{T}(\chi(t)-A(\mathbf{u}(t)-\lambda \mathbf{w}), \mathbf{w}) d t \geq 0, \quad \forall \mathbf{w} \in V
$$

therefore

$$
\int_{0}^{T}(\chi(t)-A(\mathbf{u}(t)-\lambda \mathbf{w}), \mathbf{w}) d t \geq 0, \quad \forall \mathbf{w} \in V .
$$

For $\lambda \rightarrow 0$ and using the hemicontinuity of $A$, we deduce that:

$$
\int_{0}^{T}(\chi(t)-A \mathbf{u}(t), \mathbf{w}) d t \geq 0, \quad \forall \mathbf{w} \in V
$$

whence, since $V$ is a vector space,

$$
\int_{0}^{T}(\chi(t)-A \mathbf{u}(t), \mathbf{w}) d t=0, \quad \forall \mathbf{w} \in V
$$


thus

$$
(\chi(t)-A \mathbf{u}(t), \mathbf{w}) d t \geq 0, \quad \forall \mathbf{w} \in V,
$$

therefore $\mathbf{u}(t)$ is a solution of the equation

$$
\begin{aligned}
\rho_{f}\left(\frac{\partial \mathbf{u}}{\partial t}(t), \mathbf{v}\right)+(A \mathbf{u}(t), \mathbf{v})+\rho_{f} b(\mathbf{u}(t), \mathbf{u}(t), \mathbf{v}) \\
-\rho_{f} b(w(t), \mathbf{u}(t), \mathbf{v})=\int_{\Gamma_{i n}} \mathbf{h v} d \sigma+\int_{\Gamma_{w_{1}}} \mathbf{g v} d \sigma, \quad \forall \mathbf{v} \in V
\end{aligned}
$$

and moreover

$$
\mathbf{u}(\cdot, 0)=\mathbf{u}_{0}(\cdot)
$$

with

$$
\mathbf{u} \in L^{2}(0, T ; V) \cap L^{\infty}\left(0, T ;\left[L^{2}\left(\Omega_{f}\right)\right]^{2}\right) \quad \text { and } \quad \mathbf{u}^{\prime} \in L^{1}\left(0, T ; V^{\prime}\right) .
$$

We are now able to announce the following existence theorem which ensures the existence of a weak solution of our original problem (2.4)-(2.10).

Theorem 2.8. (Existence Theorem)

Let $\mathbf{u}_{0} \in\left(L^{2}\left(\Omega_{f}\right)\right)^{2}, \mathbf{h} \in L^{2}\left(0, T ;\left(L^{2}\left(\Gamma_{i n}\right)\right)^{2}\right), \mathbf{g} \in L^{2}\left(0, T ;\left(L^{2}\left(\Gamma_{w_{1}}\right)\right)^{2}\right)$ and $\mu$ satisfying the assumptions of Lemma 1. Then there exists at least one weak solution $\mathbf{u}$ of problem (2.4)-(2.10) such that

$$
\begin{gathered}
\mathbf{u} \in L^{2}(0, T ; V) \cap L^{\infty}\left(0, T ;\left(L^{2}\left(\Omega_{f}\right)\right)^{2}\right), \\
\mathbf{u}^{\prime} \in L^{1}\left(0, T ; V^{\prime}\right) \quad\left(\mathbf{u}^{\prime}=\frac{d \mathbf{u}}{d t} \text { and } V^{\prime} \text { is the dual space of } V\right)
\end{gathered}
$$

\section{The Structure Model}

The vascular tissue ensures the transport of blood and its elasticity allows preserving a sufficient pressure so that the blood flow remains constant. The vascular wall has a very complex nature and devising an accurate model for its mechanical behaviour is rather difficult. Its structure is indeed formed by many layers with different mechanical characteristics $[6,17]$.

Being considered as a living tissue, which is essentially made of water, nonlinear isotropic and hyperelastic material properties were assumed for the structure (vessel wall, lipid core, and the fibrous cap) model. We consider in this work the St. Venant-Kirchhoff model which is a generalization of the linear model for large displacements, and is a particular case of hyperelastic materials. To describe the wall dynamics we use, as it is customary in solid mechanics, the Lagrangian description where $\Omega_{s}$ is a given material reference configuration. We describe the motion of the structure in terms of its displacement field $\eta: \Omega_{s} \times(0, T) \longrightarrow$ $\mathbb{R}^{2}$, with boundary $\partial \Omega_{s}=\Gamma_{D} \cup \Gamma_{w_{1}} \cup \Gamma_{N}$ where $\Gamma_{w_{1}}$ is the part of the boundary interacting with the fluid domain, $\Gamma_{N}$ is the part in contact with the exterior and for the sake of simplicity, we assume here, the structure to be clamped on the boundaries $\Gamma_{D}$ : Figure 3 . The differential problem for the structure part then reads 


$$
\begin{cases}\text { find } \eta=\left(\eta_{1}, \eta_{2}\right)^{T}: \Omega_{s} \longrightarrow \mathbb{R}^{2} \text { such that } & \\ \rho_{s} \frac{\partial^{2} \eta}{\partial t^{2}}-\nabla \cdot\left(\left(\mathbf{I}_{2}+\nabla \eta\right) \Sigma_{s}(\eta)\right)=0, & \text { in } \Omega_{s} \times(0, T), \\ \eta=0, & \text { on } \Gamma_{D} \times(0, T), \\ \left(\left(\mathbf{I}_{2}+\nabla \eta\right) \Sigma_{s}\right) \mathbf{n}=0, & \text { on } \Gamma_{N} \times(0, T), \\ \eta(x, 0)=\eta_{0}(x), & \text { in } \Omega_{s}, \\ \frac{\partial \eta}{\partial t}=\dot{\eta}_{0}(x), & \text { in } \Omega_{s} .\end{cases}
$$

where

$\left(\mathbf{I}_{2}+\nabla \eta\right) \Sigma_{s}$ is the first Piola-Kirchhoff tensor, $\Sigma_{s}$ the second Piola-Kirchhoff tensor, $\mathbf{I}_{2}+\nabla \eta$ is the deformation gradient tensor and $\mathbf{I}_{2}$ is the unit matrix. Moreover, we denote by $\mathbb{E}$ the Green-St. Venant strain tensor:

$$
\mathbb{E}(\eta)=\frac{1}{2}\left(\nabla \eta+(\nabla \eta)^{T}+(\nabla \eta)^{T} \nabla \eta\right) .
$$

We consider a St. Venant-Kirchhoff materiel, for which the response function for the second PiolaKirchhoff tensor is linear in $\mathbb{E}$ i.e $\Sigma_{s}=\lambda_{s} \operatorname{tr}(\mathbb{E}(\eta)) \mathbf{I}_{2}+2 \mu_{s} \mathbb{E}(\eta)$, where

$$
\lambda_{s}=\frac{\nu E}{(1-2 \nu)(1+\nu)} \text { and } \mu_{s}=\frac{E}{2(1+\nu)}
$$

are the Lamé constants, $E$ is the Young modulus and $\nu$ is the Poisson ratio.

\section{Coupling the Fluid and Structures}

Fluid-structure interaction between blood flow and wall vessel is widely studied and recently we can cite the work in [5] where the author gives a numerical approximation of the FSI problem appearing when an incompressible Newtonian fluid flows in a compliant vessel. The vessel wall dynamics is described by means of a thin elastic membrane model. In [7] the pulse wave due to the 3D interaction between blood and the vessel wall using a generalized Newtonian fluid is investigated, while in [13] a new algorithms to efficiently predict the haemodynamics in large arteries is addressed and validated. It should be noted that all these works consider a healthy wall. On the other side, fluid-structure interaction between blood flow and atherosclerotic plaque is studied in [18] where the conditions of plaque rupture in a 2D geometry are investigated, and in [11] the author proposes an incorporation of fluid-structure interaction as well as non-Newtonian effects to investigate the recirculations downstream of the plaque and the stress on the latter, but none of these studies investigate the blood-wall and blood-plaque interaction simultaneously. The coupling between the fluid and the structure occurs at the interface between both areas. Coupling conditions are imposed on the boundary $\Gamma_{w_{1}}$ : Figure 3 . It consists of a transfer operation of fields between the fluid and the structure, and concerns the transfer of compressive stress to the solid, which by deforming, transfers displacement fields to the fluid. This interaction can be described by coupling equations $(2.4),(2.5)$ with the model for structures. Two conditions are imposed on the interface:

- Continuity of velocity over time:

$$
\mathbf{u}=\frac{\partial \eta}{\partial t}, \quad \text { on } \Gamma_{w_{1}} \times(0, T)
$$

- The interface is considered in mechanical equilibrium:

$$
-\left(2 \mu(\mathbf{s}(\mathbf{u})) \mathbf{D u}-p \mathbf{I}_{2}\right) \mathbf{n}=\left(\Sigma_{s}(\eta)\right) \mathbf{n}, \quad \text { on } \Gamma_{w_{1}} \times(0, T) .
$$




\section{Numerical and Physical Parameters}

The Arbitrary Lagrangian Eulerian (ALE) formulation is particularly well suited to the study of problems with large deformations and more generally to the study of coupled problems. The choice of the ALE formulation is to use a Lagrangian framework for the solid and a mixed formulation for the fluid. The time derivatives are expressed as functions of the Lagrangian reference coordinates, whereas the space derivatives are left expressed as functions of the fixed Eulerian coordinates, since their expression is much simpler. The Lagrangian formulation of the time derivatives involves the domain velocity, which is the time derivative of the deformation function, and is often called mesh velocity since it gives the velocity of each mesh nodes in the fixed referential. The ALE formulation of the non-Newtonian equation for fluid is then

$$
\rho_{f}\left(\left.\frac{\partial \mathbf{u}}{\partial t}\right|_{X}+((\mathbf{u}-w) \cdot \nabla) \mathbf{u}\right)-\nabla_{x} \cdot(2 \mu(\mathbf{s}(\mathbf{u})) \mathbf{D u})+\nabla_{x} p=0
$$

where $X$ is the Lagrangian coordinate, $x$ the Eulerian coordinate and $w$ the mesh velocity.

We refer to [9] for a detailed description and study of the ALE method.

In this section we present some new results of numerical simulations based on the results of the theoretical study carried out in the previous sections, with some modifications in boundary conditions due to fluidstructure coupling.

The model scheme to mimic a stenosed Internal Carotid Artery (ICA) is shown in Figure 4. We have considered a rectangular domain of height $0.7 \mathrm{~cm}$ and length $L=2 \mathrm{~cm}$. Fibrous cap and lipid pool are described by cosine functions where stenosis length $l=1 \mathrm{~cm}$. The blood is modeled as a generalized Newtonian fluid defined by the Carreau law given by

$$
\mu=\mu_{\infty}+\left(\mu_{0}-\mu_{\infty}\right)\left(1+(\lambda \mathbf{s}(\mathbf{u}))^{2}\right)^{(n-1) / 2},
$$

and its flow is governed by equations $(2.4),(2.5)$ with the values of parameters taken from [1], with:

density $\rho_{f}=1060 \mathrm{Kg} \cdot \mathrm{m}^{-3}$,

viscosity for the lowest shear rates $\mu_{0}=0.0456$ Pa.s,

viscosity at higher shear rates $\mu_{\infty}=0.0032 \mathrm{~Pa} . \mathrm{s}$,

$\lambda=10.03 \mathrm{~s}$,

$n=0.344$.

In order to carry out the comparison with the Newtonian case we use $\mu=0.0035$ Pa.s. The blood flow is initially at rest and enters the vessel by the left side $\Gamma_{\text {in }}$ where the total stress on the inlet boundary has been imposed, but the amplitude of the stress varied with time and is set equal to a stress vector of magnitude $\mathbf{h}$, oriented in the negative normal direction given by the equation

$$
\mathbf{h}= \begin{cases}\left(9^{3}(1-\cos (2 \pi t / 0.005)), 0\right)^{T}, & x \in \Gamma_{i n}, 0 \mathrm{~s} \leq t \leq 0.005 \mathrm{~s}, \\ (0,0)^{T}, & x \in \Gamma_{i n}, 0.005 \mathrm{~s} \leq t<T .\end{cases}
$$

The blood flow exits the vessel by the right side $\Gamma_{\text {out }}$ where a stress-free boundary condition was imposed. The wall thickness is $0.1 \mathrm{~cm}$. The atheromatous plaque is formed of two parts: the fibrous cap and the lipid pool covered by it. These two parts are considered as hyperelastic body. The tube wall is assumed to be homogeneous, isotropic and incompressible. The structures are described as an hyperelastic material governed by equation (3.1), with:

density $\rho_{s}=1200 \mathrm{Kg} . \mathrm{m}^{-3}$,

Poisson's ratio $\nu=0.45$,

Young's Modulus $E=4 \cdot 10^{6} \mathrm{~Pa}$ for the vessel wall and for the atheromatous plaque $E=1.6 \cdot 10^{6} \mathrm{~Pa}$.

In the present study, the healthy wall $\Gamma_{w_{2}}$ is fixed and not deformed by the blood flow. No-slip boundary condition is prescribed for the fluid at this part, while the vessel wall $\Gamma_{w_{1}}$ in contact with the diseased part is not fixed, in addition to the shape of the plaque and the fluid-structures interactions between the 


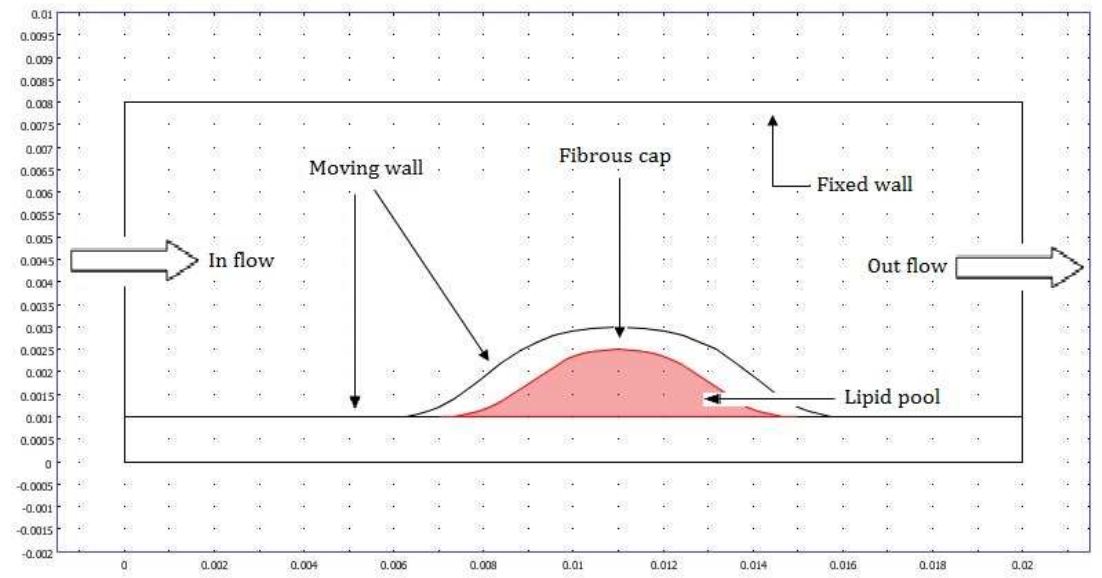

FiguRE 4. Model's geometry

flow and the vessel wall and between the flow and the plaque are investigated. The boundary conditions at the fluid-structures interfaces express kinematic and mechanical equilibria between the fluid and solids. The continuity of the velocities (4.1) and normal stresses (4.2) are imposed.

A finite element scheme was used to solve the set of motion and fluid equations using the commercial
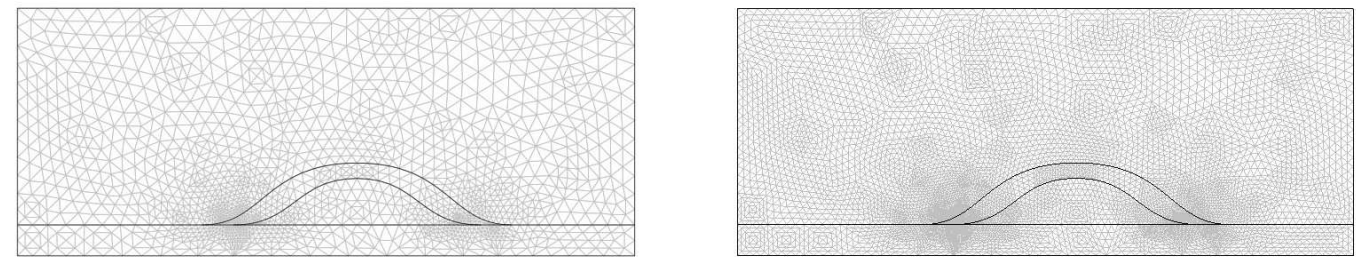

Figure 5. Typical numerical mesh showing the fluid domain, the wall, lipid core and fibrous cap. Left: 3272 elements (coarser mesh), right: 52352 elements (finer mesh)

software Comsol Multiphysics (C). The numerical meshes are depicted in Figure 5, with the fluid domain, which includes the vessel's lumen, and the solid domain, which includes the wall and lipid core. The mesh was further refined in both the solid and fluid domain around the stenosis and in regions characterized by strong gradients and elevated stresses. Mesh convergence studies in which flow parameters 


\begin{tabular}{|c|c|c|c|c||c|c|c|c|}
\hline \multirow{2}{*}{$\Delta t(\mathrm{~s})$} & \multicolumn{4}{|c||}{ Fixed Wall } & \multicolumn{4}{c|}{ Moving Wall } \\
\cline { 2 - 9 } & Newtonian Flow & non-Newtonian Flow & Newtonian Flow & non-Newtonian Flow \\
\cline { 2 - 9 } & Coarser & Finer & Coarser & Finer & Coarser & Finer & Coarser & Finer \\
\hline 0.01 & 0.234 & 0.246 & 0.234 & 0.241 & 0.247 & 0.242 & 0.236 & 0.249 \\
\hline 0.02 & 0.228 & 0.239 & 0.228 & 0.234 & 0.237 & 0.234 & 0.228 & 0.241 \\
\hline 0.05 & 0.21 & 0.218 & 0.209 & 0.215 & 0.217 & 0.215 & 0.21 & 0.22 \\
\hline 0.1 & 0.183 & 0.189 & 0.181 & 0.185 & 0.188 & 0.186 & 0.182 & 0.189 \\
\hline 0.8 & 0.0372 & 0.037 & 0.036 & 0.0359 & 0.0373 & 0.0374 & 0.0359 & 0.0359 \\
\hline
\end{tabular}

TABLE 1. Comparison of the peak velocities $(\mathrm{m} / \mathrm{s})$ for different models for meshes proposed earlier (coarser and finer) at different time (s) step.

\begin{tabular}{|c|c|c|c|c||c|c|c|c|}
\hline \multirow{2}{*}{$\Delta t(\mathrm{~s})$} & \multicolumn{4}{|c||}{ Fixed Wall } & \multicolumn{4}{c|}{ Moving Wall } \\
\cline { 2 - 9 } & Newtonian Flow & non-Newtonian Flow & Newtonian Flow & non-Newtonian Flow \\
\cline { 2 - 9 } & Coarser & Finer & Coarser & Finer & Coarser & Finer & Coarser & Finer \\
\hline 0.01 & 6.181 & 6.579 & 6.217 & 6.436 & 6.766 & 6.727 & 6.564 & 6.945 \\
\hline 0.02 & 4.912 & 5.156 & 4.97 & 5.074 & 5.412 & 5.159 & 5.122 & 5.433 \\
\hline 0.05 & 2.871 & 2.86 & 2.915 & 2.851 & 3.128 & 2.879 & 2.973 & 3.025 \\
\hline 0.1 & 1.665 & 1.579 & 1.612 & 1.322 & 1.815 & 1.288 & 1.66 & 1.496 \\
\hline 0.8 & 0.077 & 0.078 & 0.09 & 0.116 & 0.078 & 0.086 & 0.096 & 0.117 \\
\hline
\end{tabular}

TABLE 2. Comparison of the peak pressures $(\mathrm{Pa})$ for different models for meshes proposed earlier (coarser and finer) at different time (s) step.

\begin{tabular}{|c|c|c|c|c||c|c|c|c|}
\hline \multirow{2}{*}{$\Delta t(\mathrm{~s})$} & \multicolumn{4}{|c||}{ Fixed Wall } & \multicolumn{4}{c|}{ Moving Wall } \\
\cline { 2 - 9 } & Newtonian Flow & non-Newtonian Flow & Newtonian Flow & non-Newtonian Flow \\
\cline { 2 - 9 } & Coarser & Finer & Coarser & Finer & Coarser & Finer & Coarser & Finer \\
\hline 0.01 & $3.59 \mathrm{e}-7$ & $4 \mathrm{e}-7$ & $3.58 \mathrm{e}-7$ & $3.85 \mathrm{e}-7$ & $3.27 \mathrm{e}-6$ & $3.29 \mathrm{e}-6$ & $3.06 \mathrm{e}-6$ & $3.45 \mathrm{e}-6$ \\
\hline 0.02 & $3.43 \mathrm{e}-7$ & $3.83 \mathrm{e}-7$ & $3.42 \mathrm{e}-7$ & $3.68 \mathrm{e}-7$ & $3.19 \mathrm{e}-6$ & $3.24 \mathrm{e}-6$ & $2.99 \mathrm{e}-6$ & $3.40 \mathrm{e}-6$ \\
\hline 0.05 & $2.99 \mathrm{e}-7$ & $3.3 \mathrm{e}-7$ & $2.98 \mathrm{e}-7$ & $3.18 \mathrm{e}-7$ & $3.09 \mathrm{e}-6$ & $3.13 \mathrm{e}-6$ & $2.88 \mathrm{e}-6$ & $3.29 \mathrm{e}-6$ \\
\hline 0.1 & $2.14 \mathrm{e}-7$ & $2.3 \mathrm{e}-7$ & $2.14 \mathrm{e}-7$ & $2.25 \mathrm{e}-7$ & $2.44 \mathrm{e}-6$ & $2.49 \mathrm{e}-6$ & $2.32 \mathrm{e}-6$ & $2.57 \mathrm{e}-6$ \\
\hline 0.8 & $5.63 \mathrm{e}-9$ & $5.57 \mathrm{e}-9$ & $6.28 \mathrm{e}-9$ & $6.33 \mathrm{e}-9$ & $6.13 \mathrm{e}-8$ & $6.29 \mathrm{e}-8$ & $6.95 \mathrm{e}-8$ & $6.95 \mathrm{e}-8$ \\
\hline
\end{tabular}

TABLE 3. Comparison of the peak displacements $(\mathrm{m})$ for different models for meshes proposed earlier (coarser and finer) at different time (s) step.

and stress/displacement analyses with coarser and finer meshes were tested, established that the results are independent of mesh density: Table 1,2 and 3. This study is an attempt to use a theoretical model to describe the fluid velocity, global deformation, and structural shear stress. A coupled fluid-structure interaction model was used to demonstrate how blood flowed through a stenotic artery and deformed the structures. For this, an impulse input with a single sine wave over two small time periods is used [2]. The amplitude of the entrance stress has been taken equal to $1200 \mathrm{~Pa}$ which is about $1 / 8$ of the real amplitude in a cardiac beat. On the contrary the time duration of the pulse $(T=5 \mathrm{~ms})$ is much smaller than the one of a cardiac beat (systolic phase: about $0.3 \mathrm{~s}$ ). This choice has been made to amplify the propagative phenomena engendered by the fluid-structure coupling and it allows to highlight many interesting aspects both in the modelling and in the numerical approximation of the coupled problem [5], such as the differences in the flow field induced by the choice of Newtonian or non-Newtonian fluid model, and the differences in the stress/strain distribution within the structures induced by the choice of rigid or moving wall. The stress function is defined using a time step of $5 \times 10^{-3} \mathrm{~s}$ in $[0 \mathrm{~s}, 0.4 \mathrm{~s}]$ and $10^{-2}$ $\mathrm{s}$ in $[0.41 \mathrm{~s}, 0.8 \mathrm{~s}]$. The final time is $T=0.8 \mathrm{~s}$, which is the period of one heartbeat. 


\section{Numerical Results and Discussion}

In this section we present numerical results of the structural shear stress (SSS) distribution over the global system (the vessel wall and the atheromatous plaque), its displacement and blood recirculations downstream of the stenosis. Shear stress on arterial walls has been estimated in numerical models, in vitro, in animals and in humans. In many cases approximations and assumptions are necessary in order to calculate shear stress; they may thus sometimes diverge from the real flow phenomenon. Mathematical models often require a specific description of geometrical characteristics and the flow conditions of the vessel studied. The distribution of shear stresses in a vessel area can be calculated by resolving the mathematical equations describing the flow velocity in the vessel. Shear rate and viscosity are directly related to the properties of the fluid. Non-Newtonian fluids are governed by a non-linear relationship between shear stress and shear rate. In this case (non-Newtonian fluid), the slope of the shear stress-rate curve, which is equal to fluid viscosity, depends on pressure, temperature and shear rate. In contrast, the viscosity of Newtonian fluids is independent from shear rate [16]. The comparison of the maximum values of SSS for the global system between Newtonian model and non-Newtonian Carreau model in both cases, moving and fixed wall is shown in Figure 6. Note that, in the case of the fixed wall, the curves of the distribution of SSS are almost correlated with the maximum of the curve of Newtonian model that slightly exceeds the maximum of the curve of the non-Newtonian model. This joined the results already obtained in the case of blood-plaque interaction without taking into account the movement of the vessel wall, see [11]. In particular the SSS in the rigid wall simulation seems to be overestimated. Unlike the case of the moving wall, where the maximum of the curve of Newtonian model greatly exceeds the maximum of the curve of non-Newtonian model. It is to be expected because the viscosity taken in the Newtonian simulation is near the minimal value of the apparent viscosity taken in the non-Newtonian model.

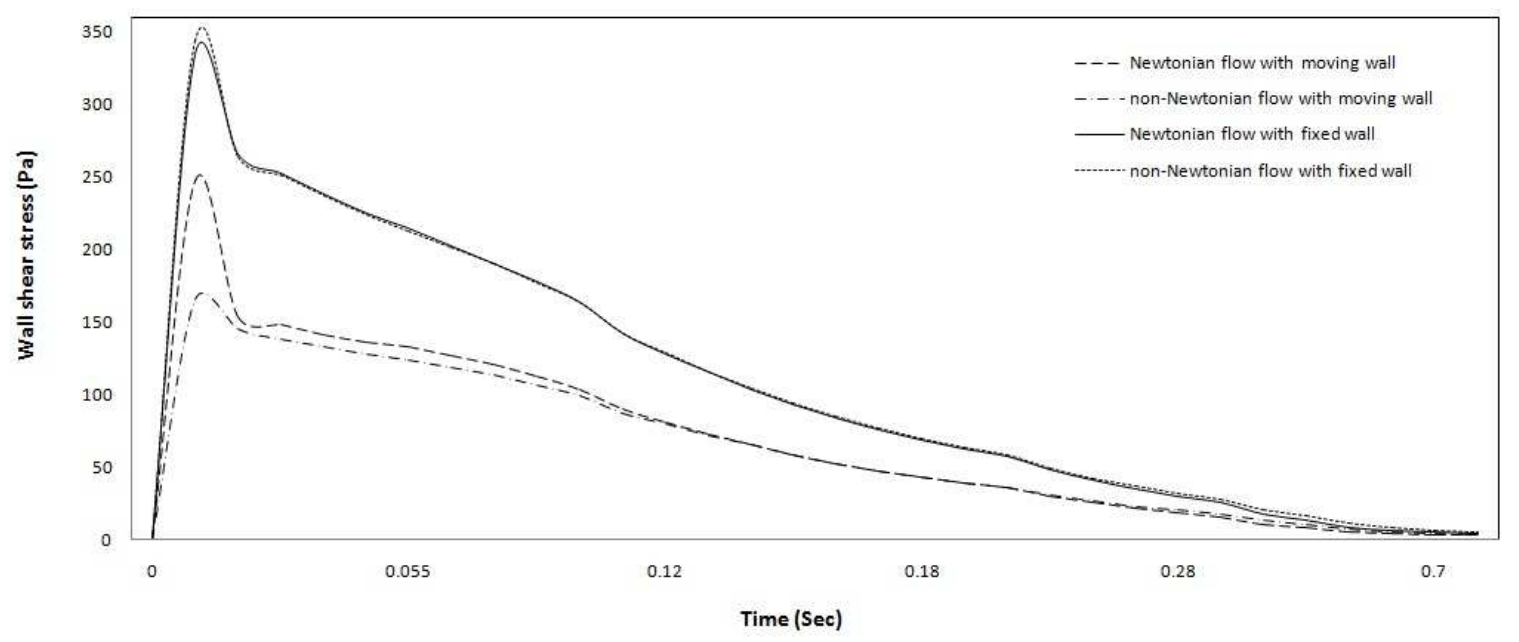

FiguRE 6. Comparison of the maximum values of SSS, for the global system in the case of fixed and moving wall, for the Newtonian model and for the non-Newtonian Carreau model.

Figure 7 shows the SSS distribution for the global system in both cases, Newtonian and non-Newtonian, as well as the deformations. This figure shows that the Newtonian fluid exerts on the plaque, a larger force. On the other hand we find a significant difference between the displacements of the system in both cases. Indeed, the Newtonian case overestimates the distribution of SSS and deformations therefore, in 
this case, it presents larger deformations and thus a greater risk of rupture. However, as shown in Figure
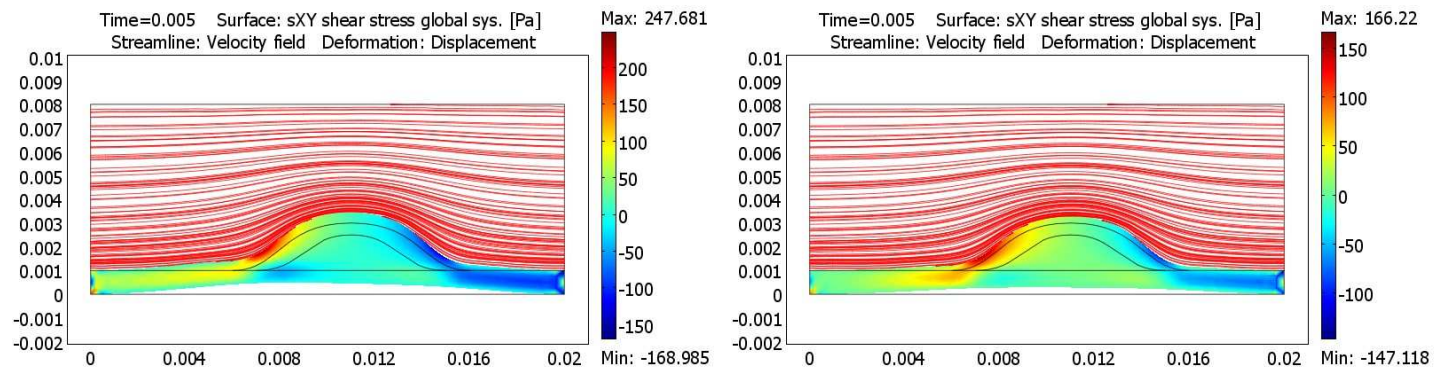

FigURE 7. SSS distribution and deformation of structures in the case of moving wall. Left: Newtonian model. Right: non-Newtonian Carreau model

8 there is, practically, no difference in the case of rigid wall. For the sake of clarity, the displacements shown in Figures 7, 8 are magnified by a factor 100.
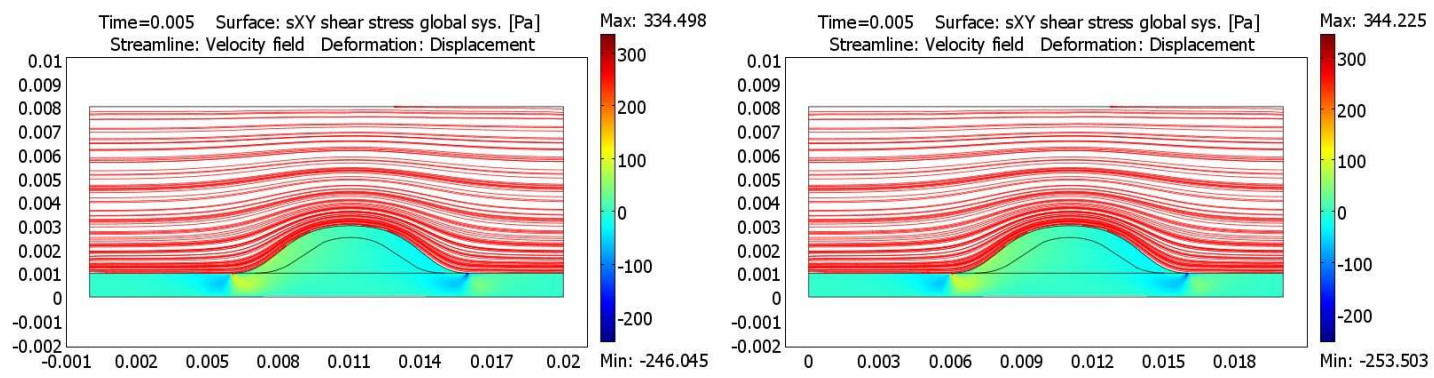

FIgURE 8. SSS distribution and deformation of structures in the case of fixed wall. Left:

Newtonian model. Right: non-Newtonian Carreau model

The other feature of interest is the recirculations over the plaque. The usual geometrical parameter to assess the risk of recirculations (and hence of blood clot formation) is the degree of stenosis, which is the proportion of arterial lumen that is occupied by the plaque. This influence of the plaque geometry is out of scope of this study, we refer to [10] and [11] for a detailed comparative study of different parameters. On the other hand we want to investigate the influence of the wall deformation on blood clot formation. In Figure 9 the blood recirculations downstream of the stenosis for non-Newtonian Carreau model are the same for both moving and fixed wall. We note that on downstream of the stenosis, the SSS is low for all cases and reverses direction because of the formation of the recirculation zone. 

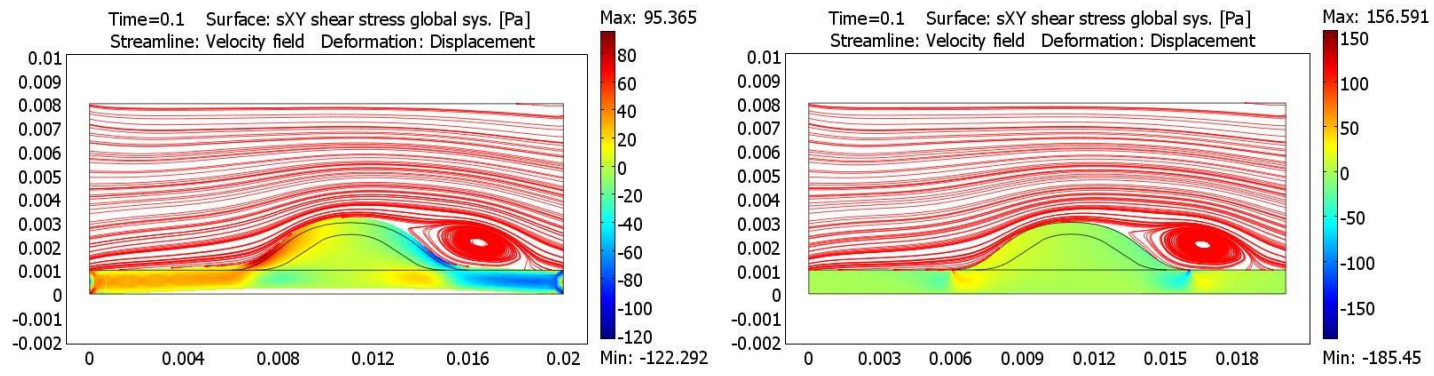

FiguRE 9. Blood recirculations downstream of the stenosis for non-Newtonian Carreau model. Left: moving wall. Right: fixed wall

\section{Conclusion}

In this work we consider a mathematical model for the interaction among the blood flow and both vessel wall and atherosclerosis plaque in a 2D idealised stenosed artery. We propose an analytical study of the generalized Navier-Stokes equations for incompressible non-Newtonian fluids with non standard boundary conditions using Faedo Galerkin method. We prove the existence of solution for the system of partial differential equations that describes the blood flow resulting from the model construction. Then, we test the model numerically by coupling it with the structure model using the ALE formulation. The parameters describing the blood rheology, the vessel wall and the plaque are taken from the literature and experimental data.

The features of interest of the numerical results are the SSS distribution over the global system, its displacement and blood recirculations downstream the stenosis. The results show that if we consider a rigid wall (without interaction with the blood) the Newtonian and non-Newtonian models overestimate the stress on the plaque. In some sense, this means that the case of fixed wall can be considered as acceptable, since it overestimates a risk. But in the case where we consider a compliant wall (with interaction with the blood) the stress over the plaque is relatively small compared to rigid wall case. Indeed, the computations show that the non-Newtonian model significantly underestimates the stress on the plaque, that is underestimating the risk of plaque rupture, which can be considered as an important drawback. The computations also show a significant difference in the displacement of the global domain. Indeed, the Newtonian model overestimates the deformation of structures. On the other hand, there is no significant difference in the blood recirculations neither between compliant and rigid wall cases nor between Newtonian and non-Newtonian ones. Thus, considering a rigid wall is acceptable but not pretentious, since the case of moving wall shows significant differences in terms of stress and displacement between Newtonian and non-Newtonian model.

A general conclusion for this paper is that considering the classical models of interaction between the Newtonian blood, the vessel wall and the atherosclerotic plaque, is less predictive of the risk of rupture because it underestimates the stress over the plaque comparing with a non-Newtonian blood.

A next step could be the modelling of these phenomena but in the case of real geometry of the blood vessel taken from medical imaging. The description of other possible models either at the mathematical analysis level or the numerical one, as well as the study of the blood flow and stress distribution for different degrees of stenoses and extension to the 3D case, will be the subject of a forthcoming work. 
Acknowledgements. This work has been supported with a grant PHC Volubilis from the French foreign office and the moroccan ministry of education and research MA/11/246 and partially supported by LIRIMA.

\section{References}

[1] A.M Gambaruto, J. Janela, A. Moura, A. Sequeira. Sensitivity of hemodynamics in a patient specific cerebral aneurysm to vascular geometry and blood rheology. Mathematical Biosciences and Engineering, 8 (2011), no. 2, 409-423.

[2] A. Quarteroni, L. Formaggia. Mathematical modelling and numerical simulation of the cardiovascular system. P.G. Ciarlet (ED.),Handbook of numerical analysis, vol XII, North-Holland, Amsterdam, (2004), 3-127.

[3] D. Cioranescu. Sur une classe de fluides non newtoniens. App. Math. and Opt, 3 (1977), no. 2/3, $263-282$.

[4] F. Hecht, G. Martin-Borret, M. Thiriet. Écoulement rhéofluidifiant dans un coude et une bifurcation plane symétrique. Application à l'écoulement sanguin dans la grande circulation. J. Phys., 3 (1996), France 6, 529-542.

[5] F. Nobile. Numerical approximation of fluid-structure interaction problems with application to haemodynamics. EPFL. $\mathrm{PhD}$ thesis, Lausanne, 2001.

[6] G. Holzapfel, T. Gasser, R. Ogden. A new constitutive framework for arterial wall mechanics and a comparative study of material models. Journal of Elasticity, 61 (2000), 1-48.

[7] J. Janela, A. Moura, A. Sequeira. A $3 D$ non-Newtonian fluid-structure interaction model for blood flow in arteries. Journal of Computational and Applied Mathematics, 234 (2010), 2783-2791.

[8] R. Temam. Navier-Stokes equations: theory and numerical analysis. Amsterdam; New York: North-Holland, 1977.

[9] L. Ait Moudid. Couplage fluide-structure pour la simulation numérique des écoulements fluides dans une conduite à parois rigides ou élastiques, en présence d'obstacles ou non. Université d'Artois. PhD thesis, Compiègne, 2007.

[10] M.X. Li, J.J. Beech-Brandt, L.R. John, P.R. Hoskins, W.J. Easson. Numerical analysis of pulsatile blood flow and vessel wall mechanics in different degrees of stenoses. Journal of Biomechanics, 40 (2007), 3715-3724.

[11] N. El Khatib. Modélisation mathématique de l'athérosclérose. Université Claude Bernard - Lyon 1. PhD thesis, Lyon, 2009.

[12] P.A. Raviart, J.M. Thomas. Introduction à l'analyse numérique des équations aux dérivées partielles. Masson, Paris, 1993.

[13] P. Crosetto, P. Raymond, S. Deparis, D. Kontaxakis, N. Stergiopulos, A. Quarteroni. Fluid-structure interaction simulations of physiological blood flow in the aorta. Computers and Fluids. Elsevier, 43 (2011), no. 1, 46-57.

[14] R. Aboulaich, S. Boujena, E. El Guarmah. A non linear diffusion model with non homogeneous boundary conditions in image restoration. Esc10 Milan, (2009), 22-26.

[15] S. Boujena. Étude d'une classe de fluides non-Newtoniens, les fluides Newtoniens généralisés. Thèse de troisième cycle. Univ. Pierre et Marrie-Currie, Paris 6, 1986.

[16] T.G. Papaioannou, Christodoulos Stefanadis. Vascular wall shear stress: basic principles and methods. Hellenic J Cardiol 46, (2005), 9-15.

[17] Y.C. Fung. Biomechanics: Mechanical properties of living tissues. Springer-Verlag, New York, 1993.

[18] Z.Y. Li, Simon P.S. Howarth, Tjun Tang, Jonathan H. Gillard. How critical is fibrous cap thickness to carotid plaque stability? A flow-plaque interaction model. Stroke 37, (2006), 1195-1196. 